\title{
On the non-parallel instability of the rotating-sphere boundary layer
}

\author{
A NTONIO SEGALINI $I^{1}$ \\ AND STEPHEN. J. GARRETT ${ }^{2}$ \\ ${ }^{1}$ Linné FLOW Centre, KTH Mechanics, 10044 Stockholm, Sweden \\ ${ }^{2}$ Department of Engineering, University of Leicester, University Road, \\ Leicester, LE1 7RH, UK \\ (Received ?; revised ?; accepted ?. - To be entered by editorial office)
}

\begin{abstract}
We present a new solution for the steady boundary-layer flow over the rotating sphere that also accounts for the eruption of the boundary layer at the equator and other higher-order viscous effects. Non-parallel corrections to the local Type I and Type II convective instability modes of this flow are also computed as a function of spin rate. Our instability results are associated with the previously observed spiral vortices and remarkable agreement between our predictions of the number of vortices and experimental observations is found. Vortices travelling at $70-80 \%$ of the local surface speed are found to be the most amplified for sufficient spin rates, also consistent with prior experimental observations.
\end{abstract}

Key words: Boundary layer; Rotating sphere; Non-parallel; Convective instability

\section{Introduction}

The flow over a rotating sphere in a quiescent fluid is present in many fields including meteorology and astrophysics, and a number of theoretical, experimental and numerical studies have been performed to investigate this flow. The slowly-rotating case was initially analytically described in the limit of infinitely small Reynolds number $\operatorname{Re}_{s}=\Omega R^{2} / \nu$ by Stokes (Dennis et al. 1980) where $\Omega, R$ and $\nu$ indicate the sphere angular velocity, the sphere radius and kinematic viscosity, respectively. Reynolds numbers of the order of $\mathcal{O}(100)$ were studied analytically by Dennis et al. (1980) by means of a series expansion in terms of Gegenbauer functions. However, the determination of the coefficients of the series expansion becomes increasingly challenging as $\operatorname{Re}_{s}$ is increased due to the growing importance of the non-linear interactions between the terms of the series. Numerical solutions of the rotating-sphere problem became recently available (see for instance Calabretto et al. 2015) but the simulation of the flow is increasingly challenging as the Reynolds number is increased, since a boundary layer is formed near the sphere surface. Instead of seeking a solution of the full Navier-Stokes equations, a feasible alternative is provided by boundary-layer theory, giving a leading description of what happens close to the surface where the angular momentum transfer takes place. The boundary-layer approximation of the rotating-sphere equations was initially proposed by Howarth (1951), while a solution of them was proposed by Banks (1965) in terms of a series expansion of the distance from the pole. However, the series diverged close to the equator, so that a numerical solution of the equations was needed after a certain latitude (Garrett \& Peake

$\dagger$ Email address for correspondence: segalini@mech.kth.se 
2002). Furthermore, as the boundary-layer equations are parabolic, they will result in a non zero azimuthal velocity at the equator, so that a collision between the two boundary layers coming from the poles must take place (Simpson \& Stewartson 1982) with a new elliptic structure of the governing equations. Anyhow, the boundary-layer equations are expected to provide a good approximation of the flow near the sphere surface and away from the equator at high Reynolds numbers but the approximation will become increasingly inaccurate for small $\operatorname{Re}_{s}$. To our knowledge, no attempt to develop a higher-order correction has been done so far.

The equatorial region deserves special attention. The experiments of Bowden \& Lord (1963); Hollerbach et al. (2002); Calabretto et al. (2015) and the numerical simulations of Dennis et al. (1980) and Dennis \& Duck (1988) suggest a more complicate structure where the two boundary layers coming from the poles collide close to the equator, generating a boundary-layer eruption that subsequently becomes a radial jet (Squire 1955; Riley 1962; Schwarz 1963). Stewartson (1958) proposed that the impinging region could be decomposed as an inviscid zone and as a very thin viscous region close to the equator plane and to the sphere surface, and described by planar Navier-Stokes equations. He did not however propose any solution for the inviscid region, despite the fact that it was described by a Poisson equation. Later, Smith \& Duck (1977) proposed an asymptotic analysis suggesting a separation zone near the corner, but this separation region has not been observed in experiments and simulations, so that the characteristics of the impinging region remain unclear.

Clearly, the boundary-layer flows over rotating disks and spheres are closely related. Specifically, the rotating sphere is locally flat close to its poles and can be thought of as corresponding to small rotating disks in those regions. A natural question is then 'how far does one have to move from a pole in order for the local sphere flow to cease sharing the stability properties of the disk?'. This question has received interest in the literature, most notably from Garrett who has shown that, like the rotating disk, the rotating sphere is locally both convectively and absolutely unstable at all latitudes for sufficiently large spin rates (Garrett \& Peake 2002, 2004; Garrett 2010; Barrow \& Garrett 2013).

The focus of continued interest in the rotating disk has, in the last two decades, moved from the study of the spiral vortices in the transitional region (Gregory et al. 1955; Hall 1986; Malik 1986), towards an attempt to explain the global behaviour of the system that results in the characteristically sharp onset of turbulence. It could be argued that this new focus began in the mid 90s with the seminal hypothesis of Lingwood (1995) that local absolute instability plays a significant role in the promotion of a global response. Some years later, Davies \& Carpenter (2003) showed by direct numerical simulations that, although the disk is indeed locally absolutely unstable, this does not lead to a linear global mode when the full non-parallel growth of the boundary layer is accounted for. That is, Lingwood's initial hypothesis may have been unduly influenced by the use of the parallel-flow approximation in her linear analysis. At around the same time Pier (2003) demonstrated explicitly that a nonlinear approach is indeed required. However, he also went on to show that Lingwood's local absolute instability fixes the onset of the non-linear global mode which, in turn, has a secondary absolute instability that promotes the transition to turbulence. This result can be viewed as confirmation of Lingwood's hypothesis but does also highlight that the rotating-disk flow is much more complicated than originally thought.

Refinements to the theoretical understanding of the precise transition mechanisms within the rotating-disk flow continue to develop with the community's access to highperformance computing and some experimental evidence supporting the latest theoretical 
predictions do now exist (the interested reader is referred to the very latest developments due to Imayama et al. 2012, 2013, 2014; Siddiqui et al. 2013; Appelquist et al. 2015b,a).

Motivated by the growing interest in the global mode over the rotating disk, Barrow et al. (2014) conducted a global stability analysis of the rotating-sphere boundary layer. Using the weakly non-parallel shear-flow formulation due to Monkewitz et al. (1993), they were able to infer the long-term global response from the strictly local absolute instability properties of the flow at each latitude. Their clear conclusion was that the sphere boundary-layer flow is in fact dictated by a linear global mode. Given the complicated non-linear nature of the rotating-disk flow and the close relationship between the two geometries, this is a very surprising result.

In light of Davies \& Carpenter's conclusions, criticisms can be levelled against Barrow et al.'s approach to the global mode and Garrett's earlier approaches to the convective instability: they rely on strictly local analyses which in turn rely on the parallel-flow approximation. This is the motivation for our present study where the parallel-flow approximation is now removed entirely from the analysis. While we present only the nonparallel convective analysis here, the opportunity is taken here to revisit the steady-flow solution over the rotating sphere. Unlike the disk, the spherical geometry is such that boundary layers originate separately in each hemisphere and travel symmetrically from each pole. The resulting collision at the equator leads to what is typically referred to as the boundary-layer eruption. All previous boundary-layer studies use the model due to Howarth (1951) and Banks (1965) in which no account is made for this eruption (and also a number of additional viscous effects are neglected) in the steady flow, and here we present a more sophisticated model. For a detailed study of the equatorial jet resulting from the eruption, see Calabretto et al. (2015).

The new steady-flow solution is presented in Section 2 and the non-parallel formulation of the stability problem detailed in Section 3. We consider the non-parallel corrections to the original convective analysis of Garrett \& Peake (2002) in Section 4. Finally, a brief discussion of the local eigenmodes is given in Section 5. Our conclusions are then drawn in Section 6.

\section{Basic flow analysis}

Let us consider an isolated steadily rotating sphere in an otherwise quiescent medium. The problem is conveniently written in a fixed reference frame $(\theta, \varphi, r)$ indicating the azimuthal, tangential and radial direction, respectively. The steady velocity field around the sphere is denoted by $\mathbf{U}=(U, V, W)$ according to the adopted reference frame. Due to the axial-symmetry of the flow, each velocity component is a function only of the radial and azimuthal position, $r$ and $\theta$, respectively. For the sake of notation, all the physical quantities will be denoted as functions of the azimuthal angle, $\theta$, and radial position, $r$, as first and second argument, respectively, and this order will be further maintained when scaled coordinates will be introduced. Here all the physical quantities are scaled by the density, $\rho$, the angular velocity, $\Omega$, and the sphere radius, $R$. The characteristic boundary-layer thickness (based on the kinematic viscosity, $\nu$ ), $\delta=(\nu / \Omega)^{1 / 2} \ll R$, is also introduced for later use together with the small perturbation parameter of the problem, namely $\epsilon=\delta / R$. The mean-flow equations are

$$
\frac{\partial U}{\partial \theta}+U \cot \theta+r \frac{\partial W}{\partial r}+2 W=0
$$




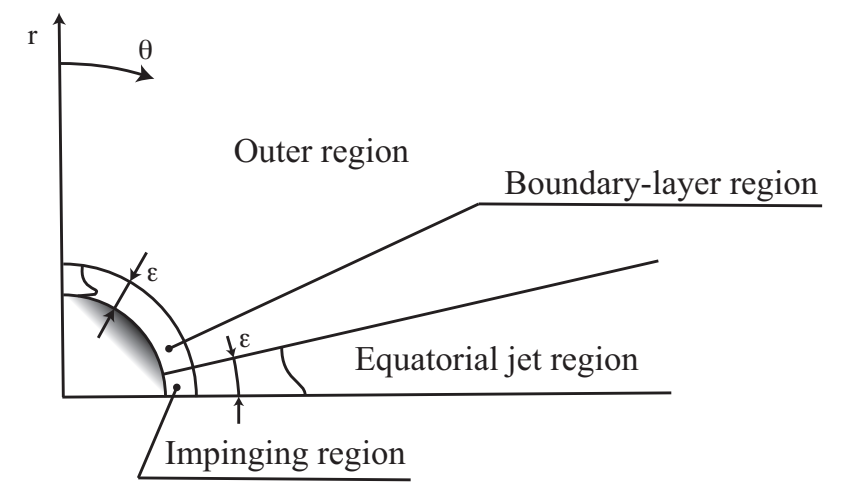

Figure 1: Schematic representation of the various regions subdividing the flow around the rotating sphere.

$$
\begin{array}{r}
\frac{U}{r} \frac{\partial \mathbf{U}}{\partial \theta}+W \frac{\partial \mathbf{U}}{\partial r}+\frac{1}{r}\left(\begin{array}{c}
U W-V^{2} \cot \theta \\
V W+U V \cot \theta \\
-U^{2}-V^{2}
\end{array}\right)=-\left(\begin{array}{c}
\frac{1}{r} \frac{\partial P}{\partial \theta} \\
0 \\
\frac{\partial P}{\partial r}
\end{array}\right)+ \\
+\frac{1}{r^{2} \operatorname{Re}_{s}}\left[\frac{\partial^{2} \mathbf{U}}{\partial \theta^{2}}+\cot \theta \frac{\partial \mathbf{U}}{\partial \theta}+r^{2} \frac{\partial^{2} \mathbf{U}}{\partial r^{2}}+2 r \frac{\partial \mathbf{U}}{\partial r}+\left(\begin{array}{c}
2 \frac{\partial W}{\partial \theta}-U / \sin ^{2} \theta \\
-V / \sin ^{2} \theta \\
-2(W+U \cot \theta)-2 \frac{\partial U}{\partial \theta}
\end{array}\right)\right]
\end{array}
$$

where $\operatorname{Re}_{s}=\Omega R^{2} / \nu=(R / \delta)^{2}=\epsilon^{-2}$ is the Reynolds number based on the sphere radius.

The base flow used for the stability calculations can be found from the solution of (2.1-2.2) but the simulations are expected to become increasingly more demanding as $\mathrm{Re}_{s}$ increases, without gaining significant insight into the scaling properties of the flow. Therefore, a boundary-layer analysis is proposed here, where the flow is subdivided as schematically illustrated in Figure 1 to make analytical progress. Historically, the analysis is usually started from the boundary layer along the sphere surface (Howarth 1951; Banks 1965). By introducing the scaled coordinate $\eta=(r-1) / \epsilon$ and the inner asymptotic sequences

$$
\begin{gathered}
U=U_{0}+\epsilon U_{1}+\mathcal{O}\left(\epsilon^{2}\right), \quad V=V_{0}+\epsilon V_{1}+\mathcal{O}\left(\epsilon^{2}\right), \\
W=\epsilon W_{0}+\epsilon^{2} W_{1}+\mathcal{O}\left(\epsilon^{3}\right) \quad, \quad P=\epsilon P_{0}+\mathcal{O}\left(\epsilon^{2}\right),
\end{gathered}
$$

it is possible to obtain the $\mathcal{O}(1)$ problem

$$
\begin{gathered}
\frac{\partial U_{0}}{\partial \theta}+U_{0} \cot \theta+\frac{\partial W_{0}}{\partial \eta}=0 \\
U_{0} \frac{\partial U_{0}}{\partial \theta}+W_{0} \frac{\partial U_{0}}{\partial \eta}-V_{0}^{2} \cot \theta=\frac{\partial^{2} U_{0}}{\partial \eta^{2}}, \\
U_{0} \frac{\partial V_{0}}{\partial \theta}+W_{0} \frac{\partial V_{0}}{\partial \eta}+U_{0} V_{0} \cot \theta=\frac{\partial^{2} V_{0}}{\partial \eta^{2}},
\end{gathered}
$$

with

and boundary conditions

$$
P_{0}(\theta, \eta)=-\int_{\eta}^{\infty}\left(U_{0}^{2}+V_{0}^{2}\right) \mathrm{d} \zeta
$$

$$
U_{0}=V_{0}-\sin \theta=W_{0}=0 \quad \text { for } \quad \eta=0,
$$


On the non-parallel instability of the rotating-sphere boundary layer

$$
U_{0}=V_{0}=\frac{\partial W_{0}}{\partial \eta}=0 \quad \text { for } \quad \eta \rightarrow \infty .
$$

The same set of equations and boundary conditions were proposed by Howarth (1951) and solved by Banks (1965) with a series of powers of $\theta$ starting from the pole. The leading term of the series is the same as the von Kármán rotating-disk flow, underlining the similarity of the two flows near the pole. However, the series solution quickly diverges for large $\theta$ and a numerical solution of the parabolic equations must instead be performed. The boundary conditions away from the wall assume that the flow outside the boundarylayer region is at rest with the exception of $W$ that has an outward velocity of order $\mathcal{O}(\epsilon)$ that triggers the flow motion outside the boundary layer. Consequently, it is expected that the velocity outside the boundary layer is $\mathcal{O}(\epsilon)$ while the pressure field is $\mathcal{O}\left(\epsilon^{2}\right)$.

The boundary-layer equations at order $\mathcal{O}(\epsilon)$ are

$$
\begin{gathered}
\frac{\partial U_{1}}{\partial \theta}+U_{1} \cot \theta+\frac{\partial W_{1}}{\partial \eta}+2 W_{0}+\eta \frac{\partial W_{0}}{\partial \eta}=0 \\
U_{0} \frac{\partial U_{1}}{\partial \theta}+U_{1} \frac{\partial U_{0}}{\partial \theta}+W_{0} \frac{\partial U_{1}}{\partial \eta}+W_{1} \frac{\partial U_{0}}{\partial \eta}+\eta W_{0} \frac{\partial U_{0}}{\partial \eta}+ \\
+U_{0} W_{0}-2 V_{0} V_{1} \cot \theta=-\frac{\partial P_{0}}{\partial \theta}+\frac{\partial^{2} U_{1}}{\partial \eta^{2}}+\eta \frac{\partial^{2} U_{0}}{\partial \eta^{2}}+2 \frac{\partial U_{0}}{\partial \eta} \\
U_{0} \frac{\partial V_{1}}{\partial \theta}+U_{1} \frac{\partial V_{0}}{\partial \theta}+W_{0} \frac{\partial V_{1}}{\partial \eta}+W_{1} \frac{\partial V_{0}}{\partial \eta}+\eta W_{0} \frac{\partial V_{0}}{\partial \eta}+V_{0} W_{0}+ \\
+\left(U_{0} V_{1}+U_{1} V_{0}\right) \cot \theta=\frac{\partial^{2} V_{1}}{\partial \eta^{2}}+\eta \frac{\partial^{2} V_{0}}{\partial \eta^{2}}+2 \frac{\partial V_{0}}{\partial \eta}
\end{gathered}
$$

The above equations are linear in the perturbation terms and are driven by the boundary conditions and by some inhomogeneous terms coming from the $\mathcal{O}(1)$ solution, including curvature effects. The associated boundary conditions are $U_{1}=V_{1}=W_{1}=0$ for $\eta=0$, while for the outer edge the conditions depend on the outer flow and will be discussed later.

Due to the parabolic nature of equations (2.4)-(2.6) and (2.10)-(2.12), the tangential velocity at the equator, $U_{0}(\pi / 2, \eta)$, will not be zero, resulting in a viscous impinging wall jet with a lateral velocity $V_{0}(\pi / 2, \eta)$. Here, the azimuthal and radial changes happen with the same aspect ratio, suggesting an elliptic nature of the equations. Following Stewartson (1958), it is possible to introduce now the scaled azimuthal position $\beta=(\theta-\pi / 2) / \epsilon$, so that the following leading-order equations are determined for the impinging region

$$
\begin{gathered}
\frac{\partial U_{I}}{\partial \beta}+\frac{\partial W_{I}}{\partial \eta}=0 \\
U_{I} \frac{\partial U_{I}}{\partial \beta}+W_{I} \frac{\partial U_{I}}{\partial \eta}=-\frac{\partial P_{I}}{\partial \beta}+\epsilon\left(\frac{\partial^{2} U_{I}}{\partial \beta^{2}}+\frac{\partial^{2} U_{I}}{\partial \eta^{2}}\right) \\
U_{I} \frac{\partial V_{I}}{\partial \beta}+W_{I} \frac{\partial V_{I}}{\partial \eta}=\epsilon\left(\frac{\partial^{2} V_{I}}{\partial \beta^{2}}+\frac{\partial^{2} V_{I}}{\partial \eta^{2}}\right) \\
U_{I} \frac{\partial W_{I}}{\partial \beta}+W_{I} \frac{\partial W_{I}}{\partial \eta}=-\frac{\partial P_{I}}{\partial \eta}+\epsilon\left(\frac{\partial^{2} W_{I}}{\partial \beta^{2}}+\frac{\partial^{2} W_{I}}{\partial \eta^{2}}\right)
\end{gathered}
$$

where the subscript $I$ indicates velocity and pressure quantities in the impinging region (see Figure 1). Differently from the boundary-layer region, all the velocity components are now $\mathcal{O}(1)$. Only the leading-order viscous terms are retained since they should generate 
new internal boundary layers along the surface of the sphere and along the equatorial plane of size $\mathcal{O}(\epsilon \sqrt{\epsilon})$ (Stewartson 1958). The associated boundary conditions are (at the leading order)

$$
\begin{gathered}
U_{I}=V_{I}-1=W_{I}=0 \quad \text { for } \quad \\
U_{I}=\frac{\partial V_{I}}{\partial \beta}=\frac{\partial W_{I}}{\partial \beta}=0 \quad \text { for } \quad \beta=0, \\
U_{I}-U_{0}(\pi / 2, \eta)=V_{I}-V_{0}(\pi / 2, \eta)=W_{I}=0 \quad \text { for } \quad \beta \rightarrow-\infty .
\end{gathered}
$$

In the region outside the boundary layers, an external inviscid flow is expected (Stewartson 1958; Smith \& Duck 1977) providing the upstream coupling needed for the elliptic region. By introducing a stream-function $\Psi_{I}$, equations (2.13)-(2.16) reduce at the leading order to

$$
\frac{\partial^{2} \Psi_{I}}{\partial \beta^{2}}+\frac{\partial^{2} \Psi_{I}}{\partial \eta^{2}}=-\omega_{I}\left(\Psi_{I}\right)
$$

with

$$
U_{I}=\frac{\partial \Psi_{I}}{\partial \eta} \quad, \quad V_{I}=V_{I}\left(\Psi_{I}\right) \quad, \quad W_{I}=-\frac{\partial \Psi_{I}}{\partial \beta},
$$

where the vorticity $\omega_{I}$ and $V_{I}$ are constants along the streamlines. The boundary conditions of $U_{I}=0$ along the sphere surface and $\partial V_{I} / \partial \beta=\partial W_{I} / \partial \beta=\omega_{I}=0$ in the equatorial plane cannot be fulfilled by (2.20) and should be satisfied through the presence of local boundary layers. The solution of (2.20) has been discussed in the literature (Rubel 1983; Phares et al. 2000), but here it is further complicated by the presence of a recirculation region where the value of the vorticity is unknown, so that equation (2.20) cannot be solved directly, but we need to solve (2.13)-(2.16) instead. Nevertheless the solution as $\eta \rightarrow \infty$ must approach the inflow, so that the outflow asymptotes to

$$
U_{I}=0 \quad, \quad V_{I}(\beta)=V_{0}(\pi / 2,-\beta) \quad, \quad W_{I}(\beta)=U_{0}(\pi / 2,-\beta) .
$$

The presence of a separation and re-attachment of the boundary layer near the equator suggests a more complex structure for the impinging region than the one proposed by Stewartson (1958). Several research works have been devoted to this region in the attempt to describe the steady flow (Stewartson 1958; Smith \& Duck 1977) as well as the transient regime (Banks \& Zaturska 1979; Simpson \& Stewartson 1982; Van Dommelen 1990): the latter studies showed evidences of a finite-time singularity in the boundary-layer equations, suggesting a structure that might escape the description achievable with simple boundary-layer theory and parabolic equations. Smith \& Duck (1977) proposed that the influence of the separation point should be felt much deeper than $\epsilon$, and they developed an analysis based upon a new scaling coordinate $\theta=\theta_{0}+\epsilon^{6 / 7} \beta^{\prime}$, where $\theta_{0}$ indicates the azimuthal position of the separation point. Some details about the upstream effect of the impinging region are proposed in the paper appendix. The result of the analysis is a correction to be applied as the equator is approached of the form given by (A 2). Such an analysis is not repeated for the re-attachment point in the equatorial plane, so that here it will be assumed that the profiles (2.22) will be recovered downstream of the re-attachment point and as $\eta \rightarrow \infty$, as suggested by Stewartson (1958).

Along the equatorial plane, radial effects will become increasingly important and the impinging-flow equations should be replaced by local parabolic equations as

$$
\frac{\partial U_{J}}{\partial \beta}+r \frac{\partial W_{J}}{\partial r}+2 W_{J}=0
$$




$$
\begin{gathered}
\frac{\partial P_{J}}{\partial \beta}=0, \\
U_{J} \frac{\partial V_{J}}{\partial \beta}+r W_{J} \frac{\partial V_{J}}{\partial r}+V_{J} W_{J}=\frac{1}{r} \frac{\partial^{2} V_{J}}{\partial \beta^{2}}, \\
U_{J} \frac{\partial W_{J}}{\partial \beta}+r W_{J} \frac{\partial W_{J}}{\partial r}-V_{J}^{2}=-r \frac{\partial P_{J}}{\partial r}+\frac{1}{r} \frac{\partial^{2} W_{J}}{\partial \beta^{2}},
\end{gathered}
$$

where $U=\epsilon U_{J}$ and $V_{J}, W_{J}=\mathcal{O}(1)$. The boundary conditions for the equatorial region are

$$
\begin{gathered}
U_{J}=\frac{\partial V_{J}}{\partial \beta}=\frac{\partial W_{J}}{\partial \beta}=0 \quad \text { for } \quad \beta=0, \\
\frac{\partial U_{J}}{\partial \beta}=V_{J}=W_{J}=0 \quad \text { for } \quad \beta \rightarrow-\infty .
\end{gathered}
$$

Since the external pressure is of order $\mathcal{O}\left(\epsilon^{2}\right)$, the pressure gradient can be removed from the analysis. Stewartson (1958) suggested that this region could be described as a radial jet (Squire 1955; Schwarz 1963), but the initial developing region differs due to the transfer of momentum between the radial and tangential velocity. A self-similar solution is possible in the limit of $r \rightarrow \infty$ with the assumption that the tangential velocity vanishes faster than the radial one. The self-similar solution is given by

$$
U_{J}=-\frac{\sqrt{2}}{\bar{\delta} r} \tanh \left(\frac{\xi}{\sqrt{2}}\right) \quad, \quad W_{J}=\frac{1}{\bar{\delta}^{2} r} \operatorname{sech}^{2}\left(\frac{\xi}{\sqrt{2}}\right),
$$

with $\xi=\beta / \bar{\delta}$ (Riley 1962; Calabretto et al. 2015). It should be noted that the characteristic variable $\xi$ is constant in the radial direction $\dagger$. Differently from in the radial-jet case, the presence of the tangential velocity in the equations leads to a transfer of momentum between the $V$ and $W$ components, so that $\bar{\delta}$ cannot be assessed from the initial available momentum integral but rather as a result of the numerical simulation of equations (2.23)-(2.26) leading to $\bar{\delta}=1.5372$. In both the numerical and self-similar solutions, the tangential velocity $\epsilon U_{J}(\beta \rightarrow-\infty, r)$ is $\mathcal{O}(\epsilon)$ and must be balanced in the outer layer.

As evident from the previous analyses, the flow outside the sphere boundary layer is inviscid and driven by the wall-normal velocity, $\epsilon W_{0}(\theta, \eta \rightarrow \infty)$, and the radial velocity outside of the equatorial radial jet, $\epsilon U_{J}(\beta \rightarrow-\infty, r)$, and indeed the velocity field in the outer layer is of order $\mathcal{O}(\epsilon)$. These ejection velocities are normal to the surface and do not carry tangential momentum, so that $V=0$ in the outer flow. Furthermore, the vorticity outside the boundary layer and radial jet is zero, so that the outside region is irrotational. It is possible to introduce a Stokes stream-function, $\Psi_{O}(\theta, r)$, so that the velocity components are described by

$$
U_{O}=-\frac{1}{r \sin \theta} \frac{\partial \Psi_{O}}{\partial r} \quad, \quad W_{O}=\frac{1}{r^{2} \sin \theta} \frac{\partial \Psi_{O}}{\partial \theta} .
$$

Since the flow is irrotational, the equation that describes the outer region is

$$
\frac{\partial^{2} \Psi_{O}}{\partial r^{2}}+\frac{1}{r^{2}} \frac{\partial^{2} \Psi_{O}}{\partial \theta^{2}}-\frac{\cot \theta}{r^{2}} \frac{\partial \Psi_{O}}{\partial \theta}=0
$$

$\dagger$ This is only apparently against the conjecture of Stewartson (1958): this apparent inconsistency is due to the different reference frames used as the thickness of the jet is constant in the $\beta-r$ plane (since $\beta$ is an angle), so that the jet will linearly expand in thickness. 
subjected to the boundary conditions

$$
\begin{array}{r}
\Psi_{O}(0, r)=0 \quad, \quad \Psi_{O}(\theta, 1)=\int_{0}^{\theta} \sin \chi W_{B}(\chi, \eta \rightarrow \infty) \mathrm{d} \chi \\
\Psi_{O}\left(\frac{\pi}{2}, r\right)=\int_{0}^{\pi / 2} \sin \chi W_{0}(\chi, \eta \rightarrow \infty) \mathrm{d} \chi-\int_{1}^{r} \chi U_{J}(\beta \rightarrow-\infty, \chi) \mathrm{d} \chi \\
\frac{\partial^{2} \Psi_{O}}{\partial r^{2}}=0 \quad \text { as } \quad r \rightarrow \infty .
\end{array}
$$

The solution of problem (2.31), together with the boundary conditions (2.32), provides the external flow field needed to create the higher-order correction for the sphere boundary layer and equatorial jet. In particular, for the former, the outer-edge boundary conditions can be determined for the $\mathcal{O}(\epsilon)$ problem $(2.10)-(2.12)$ as

$$
U_{1}(\theta, \eta)-U_{O}(\theta, 0)=V_{1}=0 \quad \text { for } \quad \eta \rightarrow \infty,
$$

together with the continuity equation enforced to determine $\partial W_{1} / \partial \eta$ at the outer edge.

\subsection{Numerical solution of the mean-flow equations}

To obtain the mean flow at the leading order, the boundary-layer equations (2.4)-(2.6) had to be solved first. These will provide the boundary-layer flow near the sphere surface and away from the impinging region (namely within a region $0 \leqslant \theta<\pi / 2-\mathcal{O}(\epsilon)$ ). Similarly to Garrett \& Peake (2002), the Banks (1965) solution (here extended to the $15^{\text {th }}$ order) is used until $\theta \approx 6^{\circ}$, beyond which a finite-difference solution of the boundarylayer equations is proposed. This is based on a Newton method combined with a spacemarching technique, significantly decreasing the computational time. The convergence criterion for the Newton method, implemented here and in the radial-jet flow, was imposed such that the correction velocity should be less than $10^{-5}$ of the equatorial tangential velocity to exit the iterative loop. A second-order central finite-difference scheme was used to discretise the derivatives in the wall-normal direction, while a second-order backward scheme was adopted for the $\theta$ derivatives. The domain was discretised into 300 grid points uniformly distributed in the wall-normal direction between the sphere surface and $\eta_{\max }=30$, and 1500 uniformly distributed points between the pole and the equator. Figure 2 shows some of the obtained velocity profiles along the sphere surface. The wall jet in the azimuthal velocity is slowly established and tends to decelerate and rise as the equator is approached. On the other hand, both the radial and tangential velocities show instead a monotonic trend.

After the impinging region, the equatorial jet takes place. Here the parabolic equations are solved again with a space-marching method based on a Newton iterative approach: a first-order backward differentiation scheme is used in the radial direction, while a central second-order scheme is used in the azimuthal direction. The latter is discretised in 200 grid points uniformly spaced between 0 and $\beta_{\max }=20$, while the radial domain is discretised in 200 grid points logarithmically spaced between $r_{\min }=1.001$ (where the inlet condition is imposed) and $r_{\max }=20$. As proposed by Stewartson (1958), the outlet of the impinging region has the same shape as the inlet, although the symmetry boundary condition is not fulfilled. A sudden transient is then expected to adjust for the boundary condition, but at $r=1.1$ the transient is terminated and the jet decays as expected (see Figure 3 ). The tangential velocity decays faster than the radial one, allowing for the self-similar solution (2.29).

The outer region is solved with a point Gauss-Seidel method based on second-order central finite differences in both the radial and azimuthal directions discretised with 1000 

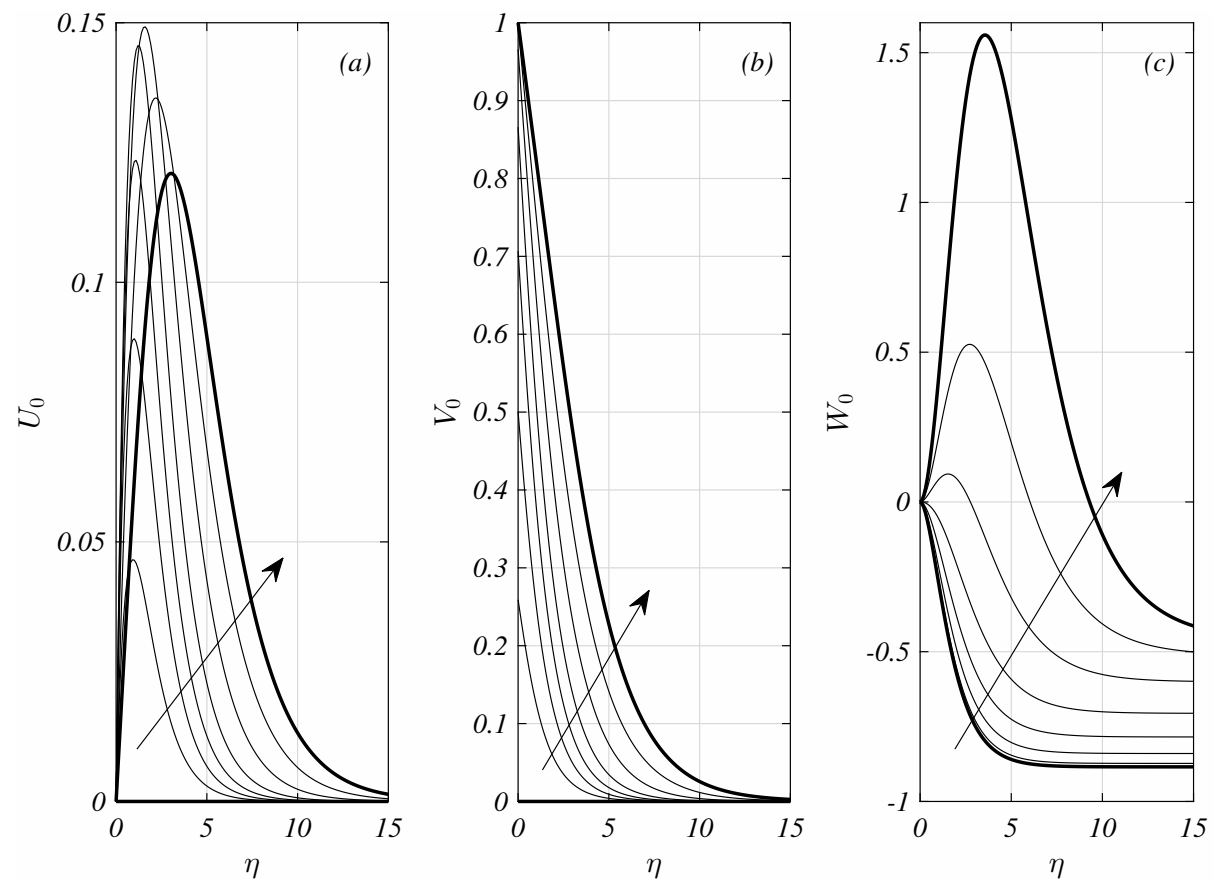

Figure 2: Sphere boundary-layer velocity profiles from the numerical solution of (2.4)(2.6) for $\theta=0^{\circ}, 15^{\circ}, 30^{\circ}, 45^{\circ}, 60^{\circ}, 75^{\circ}, 80^{\circ}, 85^{\circ}, 90^{\circ}$ according to the arrows' direction. The profiles at $\theta=0^{\circ}$ and $\theta=90^{\circ}$ are marked by thick lines.

and 500 grid points, respectively. The outermost boundary was set again to $r_{\max }=20$. Figure 4 shows the blowing velocities outside of the sphere boundary layer and of the equatorial jet. These drive the potential flow outside and generate the streamline pattern shown in Figure 5.

From the outer stream-function, the first-order perturbation velocity for the outer part of the sphere boundary layer can be determined (here shown in Figure 4a). The latter provides the outer boundary condition for the first-order problem that can be solved with the same numerical techniques used in the $\mathcal{O}(1)$ problem (with the addition of some inhomogeneous terms) providing the first-order correction to the Howarth (1951) theory. Figure 6 shows a collection of profiles of the first-order problem at the same azimuthal positions (except $90^{\circ}$ ) used in Figure 2. Qualitatively, the correction implies a speed-up of the radial jet and a decrease of the tangential velocity diffusion.

In order to validate the predictions of the sphere boundary-layer velocity profiles from the perturbation theory, a finite-difference solution of the full equations (2.1-2.2) was performed. The numerical code solved the transformed equations proposed by Dennis et al. (1980) with a point Gauss-Seidel technique and second-order central finite differences to discretise the spatial derivatives. The domain was discretised into 300 grid points in the azimuthal direction and 200 in the $r^{-1}$ space between the sphere surface and $r_{\max }=\infty$. A comparison between the finite-difference solution and the boundary-layer solution (for both the $\mathcal{O}(1)$ and the corrected one) is reported for three Reynolds numbers in Figures 7-9. The corrected profiles include both the $\mathcal{O}(\epsilon)$ correction due to the Reynolds number effects and the $\mathcal{O}\left(\epsilon^{6 / 7}\right)$ correction near the equator. As is visible from the figure, the corrections improve the agreement between the predicted boundary-layer velocity profiles and the simulation results, especially when the Reynolds number is low 
10

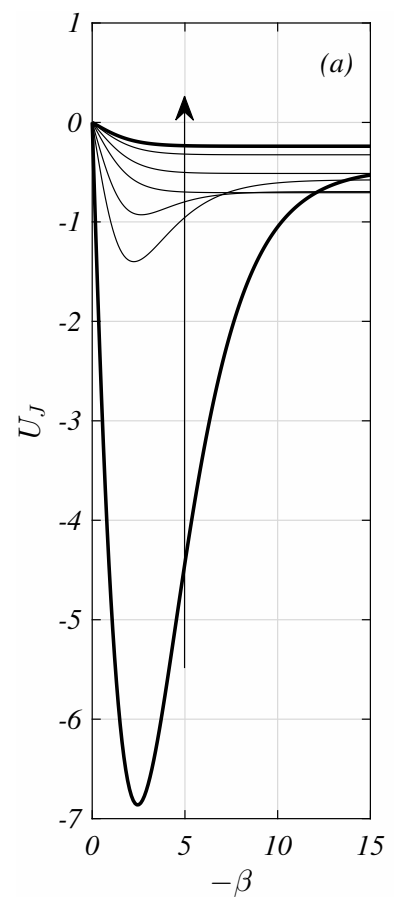

A. Segalini and S.J. Garrett

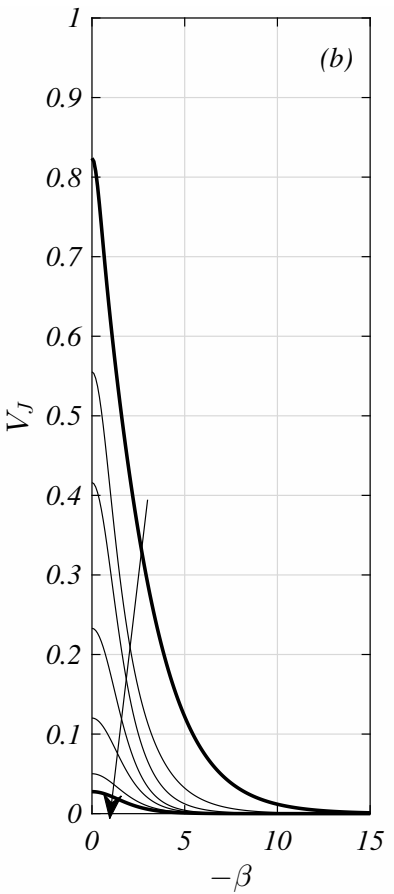

Figure 3: Equatorial jet velocity profiles from the numerical solution of (2.23)-(2.26) for $r=1.01,1.1,1.2,1.5,2,3,4$ according to the arrows' direction. The profiles at $r=1.01$ and $r=4$ are marked by thick lines.
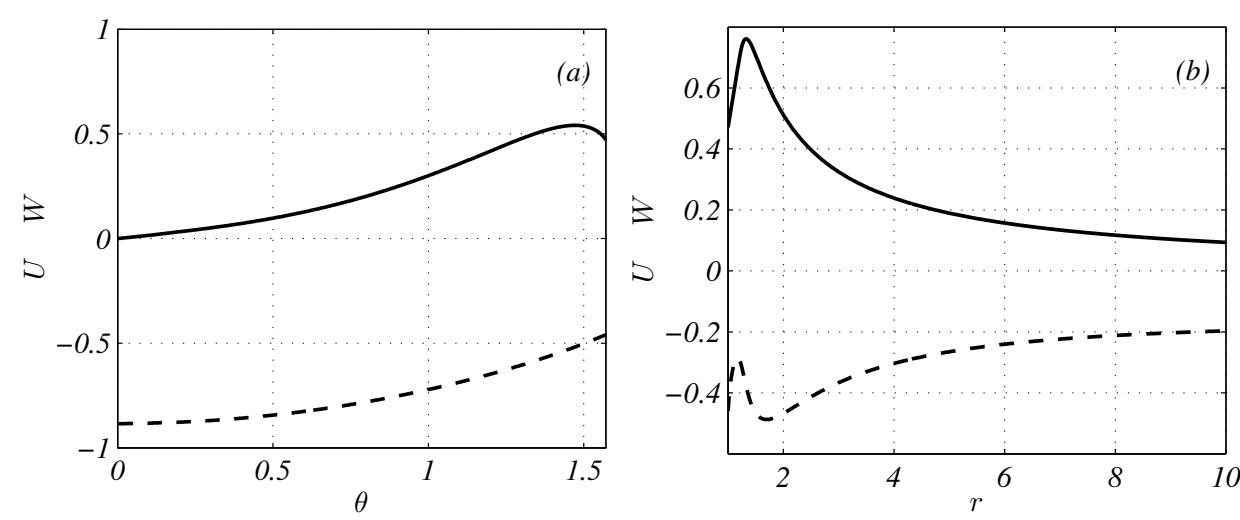

Figure 4: Outer region velocities above the sphere boundary layer $(a)$ and above the equatorial plane $(b)$. Solid lines indicate $U$, while dashed lines indicate $W$.

and when the azimuth approaches $\theta=\pi / 2$. The corrections are not able to deal properly with the region near the equator and therefore it fails there, limiting its use to a range $0 \leqslant \theta \leqslant \pi / 2-\mathcal{O}(\epsilon)$.

It is interesting to consider the evolution of the equatorial jet velocity field to assess whether the approximation regarding its inlet velocity distribution (given by 2.22) affected its estimation. Figures 10 and 11 show the distribution of the radial and tangential velocity components along the equatorial plane. It is evident that a small disagreement 


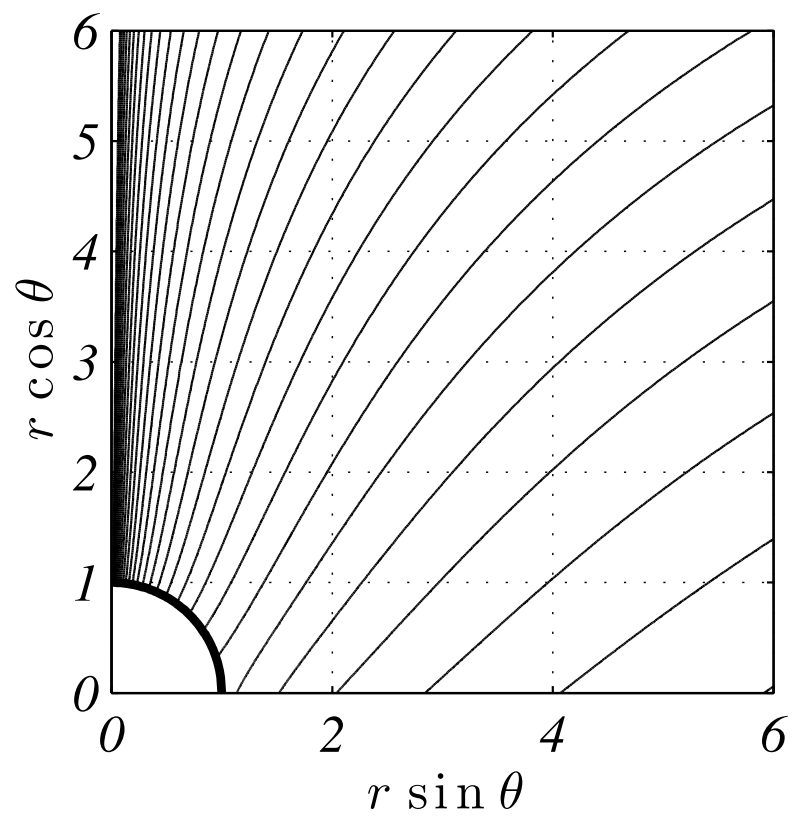

Figure 5: Iso-contours of the outer stream-function $\Psi_{O}$.
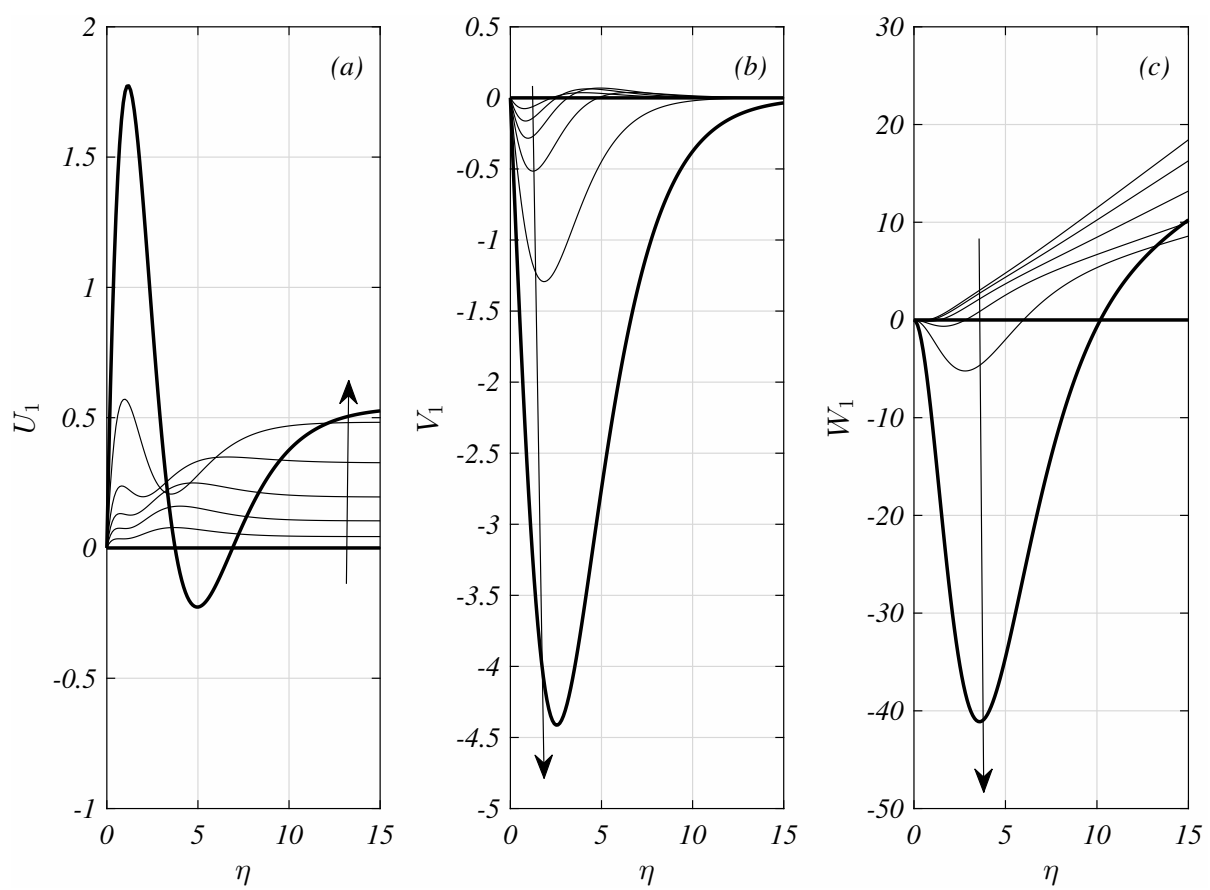

Figure 6: Boundary-layer velocity profiles from the numerical solution of (2.10)-(2.12) for $\theta=0^{\circ}, 15^{\circ}, 30^{\circ}, 45^{\circ}, 60^{\circ}, 75^{\circ}, 80^{\circ}, 85^{\circ}$ according to the arrows' direction. The profiles at $\theta=0^{\circ}$ and $\theta=85^{\circ}$ are marked by thick lines. 

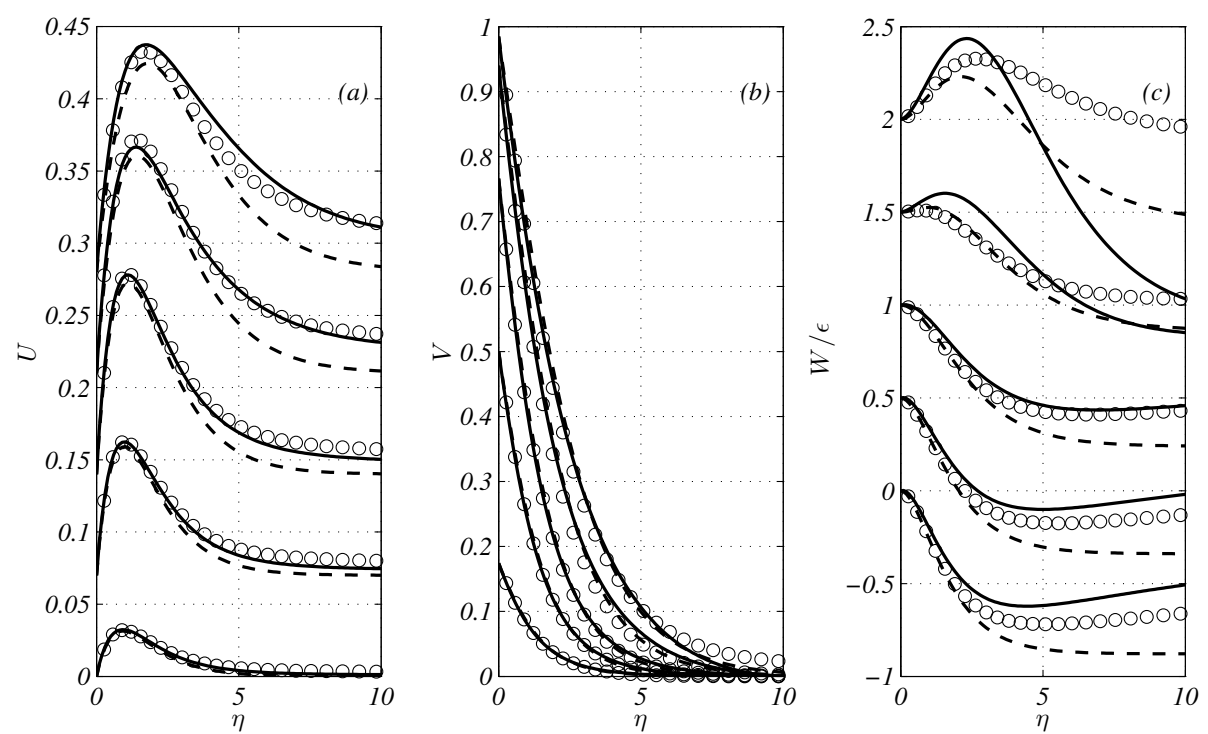

Figure 7: Comparison of the azimuthal $(a)$, tangential $(b)$ and radial $(c)$ velocities near the sphere surface for $\theta=10^{\circ}, 30^{\circ}, 50^{\circ}, 70^{\circ}, 80^{\circ}$ and $\operatorname{Re}_{s}=500$. (circles) Numerical solution of the full equations, (dashed line) solution of the boundary-layer equations (2.4-2.6), (solid line) perturbed boundary-layer solution.
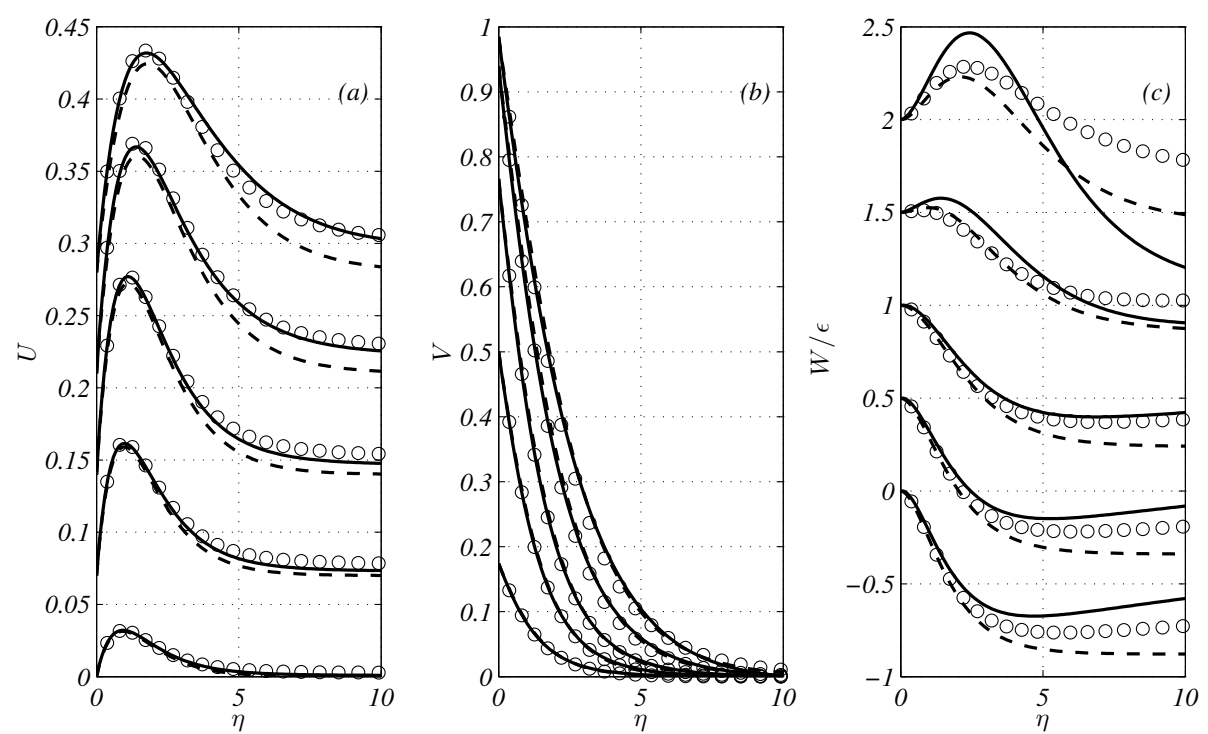

Figure 8: Comparison of the azimuthal (a), tangential $(b)$ and radial $(c)$ velocities near the sphere surface for $\theta=10^{\circ}, 30^{\circ}, 50^{\circ}, 70^{\circ}, 80^{\circ}$ and $\operatorname{Re}_{s}=1000$. See Figure 7 for the list of the used symbols.

is present near the sphere surface (mostly due to the absence of the boundary-layer analysis), but the same decay is quickly established and the jet solution represents indeed the outer limit when $\operatorname{Re}_{s} \rightarrow \infty$. Interestingly, the decay rate of the radial velocity goes 

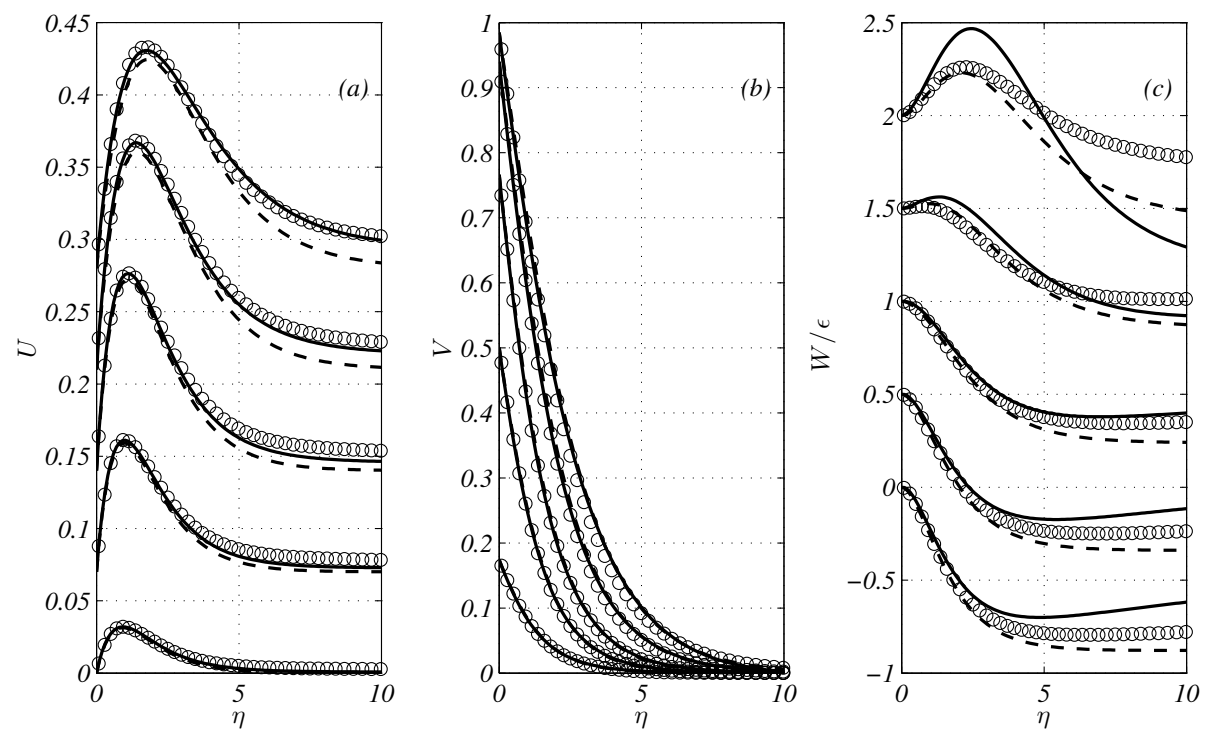

Figure 9: Comparison of the azimuthal $(a)$, tangential $(b)$ and radial $(c)$ velocities near the sphere surface for $\theta=10^{\circ}, 30^{\circ}, 50^{\circ}, 70^{\circ}, 80^{\circ}$ and $\operatorname{Re}_{s}=1500$. See Figure 7 for the list of the used symbols.

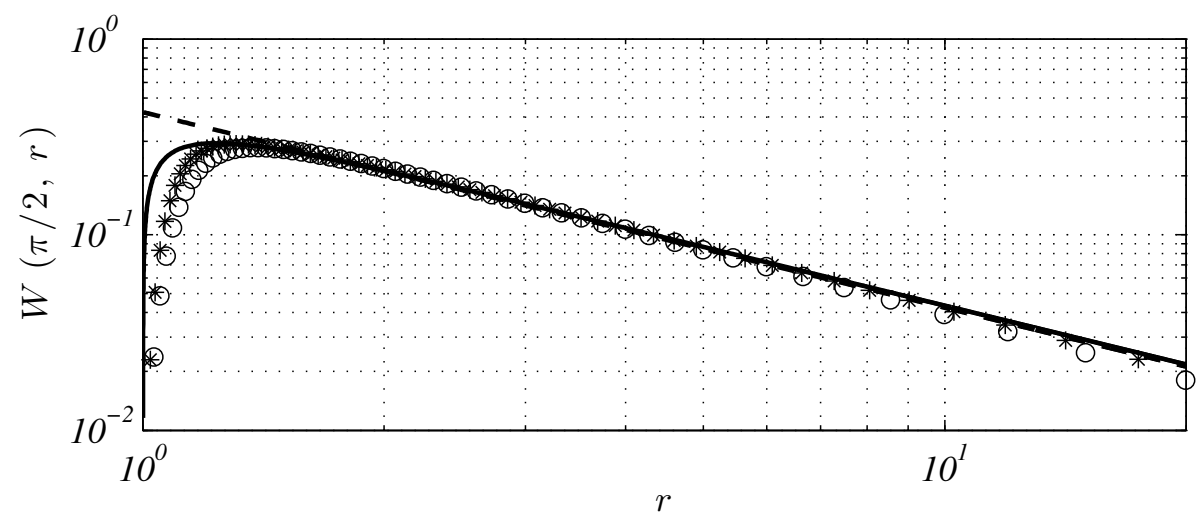

Figure 10: Radial velocity along the equatorial plane estimated from the numerical simulations at $\operatorname{Re}_{s}=500$ (circles) and $\operatorname{Re}_{s}=1500$ (asterisks). (Solid line) $W_{J}(0, r)$ as solution of equations (2.23-2.26). (Dashed line) self-similar expression (2.29).

as $r^{-1}$ in agreement with the similarity solution (2.29), while the tangential velocity is faster and goes as $r^{-2}$ following the similarity solution of Riley (1962).

\section{Formulation of the stability analysis}

Let us consider now a small perturbation to the base state $(\mathbf{u}, p)$ with $\mathbf{u}=(u, v, w)$ indicating the velocity perturbation and $p$ the pressure perturbation that can vary in time and space. By assuming the perturbation to be small, the continuity and linearised Navier-Stokes equations can be written. After subtracting the corresponding steady equa- 


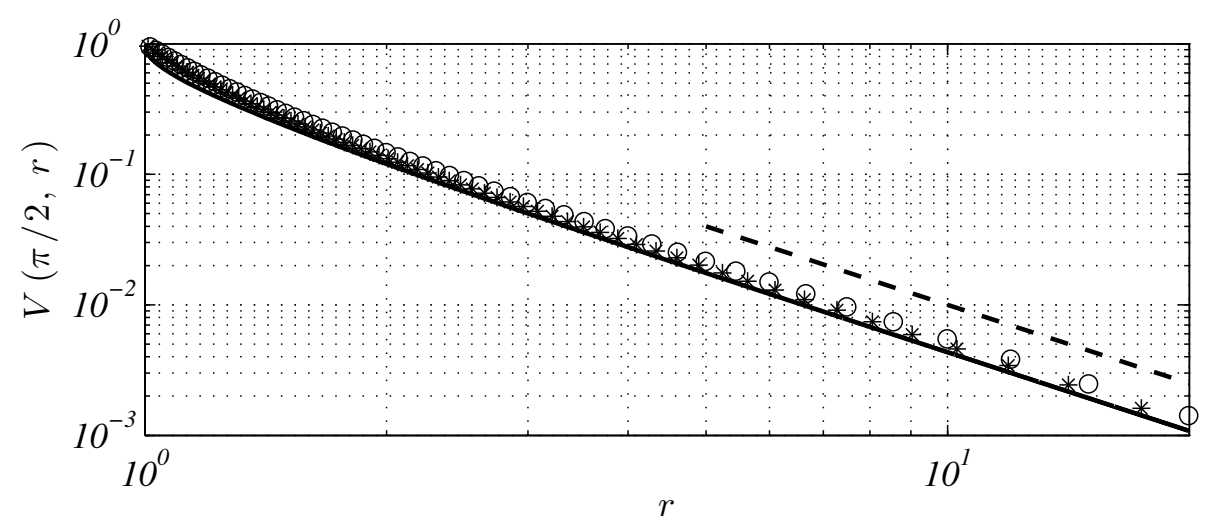

Figure 11: Tangential velocity along the equatorial plane estimated from the numerical simulations at $\operatorname{Re}_{s}=500$ (circles) and $\operatorname{Re}_{s}=1500$ (asterisks). (Solid line) $V_{J}(0, r)$ as solution of equations (2.23)-(2.26). (Dashed line) line proportional to $r^{-2}$.

tions for the mean flow, it is possible to obtain the equations governing the perturbations

$$
\begin{gathered}
\frac{\partial u}{\partial \theta}+\cot \theta u+\frac{1}{\sin \theta} \frac{\partial v}{\partial \varphi}+r \frac{\partial w}{\partial r}+2 w=0, \\
\frac{\partial \mathbf{u}}{\partial t}+(\mathbf{U} \cdot \nabla) \mathbf{u}+(\mathbf{u} \cdot \nabla) \mathbf{U}=-\nabla p+\frac{1}{\operatorname{Re}_{s}} \nabla^{2} \mathbf{u},
\end{gathered}
$$

where the various terms are given classically (Kundu et al. 2016) by

$$
\begin{aligned}
& (\mathbf{U} \cdot \nabla) \mathbf{u}=\frac{U}{r} \frac{\partial \mathbf{u}}{\partial \theta}+\frac{V}{r \sin \theta} \frac{\partial \mathbf{u}}{\partial \varphi}+W \frac{\partial \mathbf{u}}{\partial r}+\frac{1}{r}\left(\begin{array}{c}
U w-V \cot \theta v \\
V w+V \cot \theta u \\
-U u-V v
\end{array}\right) \\
& (\mathbf{u} \cdot \nabla) \mathbf{U}=\frac{u}{r} \frac{\partial \mathbf{U}}{\partial \theta}+w \frac{\partial \mathbf{U}}{\partial r}+\frac{1}{r}\left(\begin{array}{c}
W u-V \cot \theta v \\
W v+U \cot \theta v \\
-U u-V v
\end{array}\right) \\
& \nabla p=\left(\frac{1}{r} \frac{\partial p}{\partial \theta}, \frac{1}{r \sin \theta} \frac{\partial p}{\partial \varphi}, \frac{\partial p}{\partial r}\right)^{T} \\
& \nabla^{2} \mathbf{u}=\frac{1}{r^{2}}\left\{\frac{\partial^{2} \mathbf{u}}{\partial \theta^{2}}+\cot \theta \frac{\partial \mathbf{u}}{\partial \theta}+\frac{1}{\sin ^{2} \theta} \frac{\partial^{2} \mathbf{u}}{\partial \varphi^{2}}+r^{2} \frac{\partial^{2} \mathbf{u}}{\partial r^{2}}+2 r \frac{\partial \mathbf{u}}{\partial r}+\right. \\
& +\left(-\frac{u}{\sin ^{2} \theta}+2 \frac{\partial w}{\partial \theta}-\frac{2 \cot \theta}{\sin \theta} \frac{\partial v}{\partial \varphi},-\frac{v}{\sin ^{2} \theta}+\frac{2}{\sin \theta} \frac{\partial w}{\partial \varphi}+\frac{2 \cot \theta}{\sin \theta} \frac{\partial u}{\partial \varphi},\right. \\
& \left.\left.-2(w+\cot \theta u)-2 \frac{\partial u}{\partial \theta}-\frac{2}{\sin \theta} \frac{\partial v}{\partial \varphi}\right)^{T}\right\} .
\end{aligned}
$$

As our primary interest lies in the near-wall region, the same stretched wall-normal coordinate, $\eta$, is introduced as in Section 2. Furthermore, as the radial velocity is $\mathcal{O}(\epsilon)$ compared to the azimuthal and tangential ones, the notation $W=\epsilon \bar{W}$ will be adopted for the mean wall-normal velocity, which results in a balanced continuity equation.

At this point, the modal assumption for the velocity and pressure perturbations is 
introduced in the form (Monkewitz et al. 1993; Barrow et al. 2014)

$$
\phi(\theta, \varphi, \eta, t)=\hat{\phi}(\theta, \eta) \exp \left\{\frac{i}{\epsilon}\left[n \varphi-\omega t+\int_{\theta_{0}}^{\theta} \alpha(\xi) \mathrm{d} \xi\right]\right\}
$$

where $n / \epsilon$ must be an integer number in order to ensure the periodicity in the tangential direction. It is illustrative to look at the continuity equation for the perturbation quantities

$$
\frac{1}{\epsilon}\left(i \alpha \hat{u}+\frac{i n}{\sin \theta} \hat{v}+\frac{\partial \hat{w}}{\partial \eta}\right)+\frac{\partial \hat{u}}{\partial \theta}+\cot \theta \hat{u}+\eta \frac{\partial \hat{w}}{\partial \eta}+2 \hat{w}=0 .
$$

By assuming a perturbation expansion of the form $\hat{\phi}(\theta, \eta)=\hat{\phi}_{0}+\epsilon \hat{\phi}_{1}+\mathcal{O}\left(\epsilon^{2}\right)$, Eq. (3.8) leads to the problems at the orders $\mathcal{O}\left(\epsilon^{-1}\right)$ and $\mathcal{O}(1)$

$$
\begin{gathered}
i \alpha \hat{u}_{0}+i \beta \hat{v}_{0}+\frac{\partial \hat{w}_{0}}{\partial \eta}=0 \quad \text { with } \quad \beta=\frac{n}{\sin \theta}, \\
i \alpha \hat{u}_{1}+i \beta \hat{v}_{1}+\frac{\partial \hat{w}_{1}}{\partial \eta}=-\left(\frac{\partial \hat{u}_{0}}{\partial \theta}+\cot \theta \hat{u}_{0}+\eta \frac{\partial \hat{w}_{0}}{\partial \eta}+2 \hat{w}_{0}\right) .
\end{gathered}
$$

By introducing the state vector $\mathbf{q}=(\hat{u}, \hat{v}, \hat{w}, \hat{p})^{T}$, it is possible to write down the stability equations at the orders $\mathcal{O}\left(\epsilon^{-1}\right)$ and $\mathcal{O}(1)$ as

$$
\mathcal{L} \mathbf{q}_{0}=\mathbf{0}
$$

$$
\mathcal{L} \mathbf{q}_{1}=-\mathcal{H} \mathbf{q}_{0},
$$

where the operators $\mathcal{L}$ and $\mathcal{H}$ are given by

$$
\begin{aligned}
& \mathcal{L}=\left[-i \omega+i \alpha U+i \beta V-\frac{1}{\epsilon \operatorname{Re}_{s}}\left(\frac{\partial^{2}}{\partial \eta^{2}}-\alpha^{2}-\beta^{2}\right)\right] \mathbf{Q}+\left(\begin{array}{cccc}
0 & 0 & \frac{\partial U}{\partial \eta} & i \alpha \\
0 & 0 & \frac{\partial V}{\partial \eta} & i \beta \\
0 & 0 & 0 & \frac{\partial}{\partial \eta} \\
i \alpha & i \beta & \frac{\partial}{\partial \eta} & 0
\end{array}\right) \\
& \mathcal{H}=\left[i \eta(-2 \omega+\alpha U+\beta V)+U \frac{\partial}{\partial \theta}+\bar{W} \frac{\partial}{\partial \eta}-\right. \\
& \left.-\frac{1}{\epsilon \operatorname{Re}_{s}}\left(2 i \alpha \frac{\partial}{\partial \theta}+i \frac{\partial \alpha}{\partial \theta}+i \alpha \cot \theta+2 \eta \frac{\partial^{2}}{\partial \eta^{2}}+2 \frac{\partial}{\partial \eta}\right)\right] \mathbf{Q}+ \\
& +\left(\begin{array}{cccc}
\frac{\partial U}{\partial \theta} & -2 V \cot \theta & 2 \eta \frac{\partial U}{\partial \eta}+U & i \eta \alpha+\frac{\partial}{\partial \theta} \\
\frac{\partial V}{\partial \theta}+V \cot \theta & U \cot \theta & 2 \eta \frac{\partial V}{\partial \eta}+V & i \eta \beta \\
-2 U & -2 V & \frac{\partial W}{\partial \eta} & 2 \eta \frac{\partial}{\partial \eta} \\
\cot \theta+\frac{\partial}{\partial \theta} & 0 & \eta \frac{\partial}{\partial \eta}+2 & 0
\end{array}\right)- \\
& -\frac{2 i}{\epsilon \operatorname{Re}_{s}}\left(\begin{array}{cccc}
0 & -\beta \cot \theta & \alpha & 0 \\
\beta \cot \theta & 0 & \beta & 0 \\
-\alpha & -\beta & 0 & 0 \\
0 & 0 & 0 & 0
\end{array}\right)
\end{aligned}
$$


and the matrix $\mathbf{Q}$ defined as

$$
\mathbf{Q}=\left(\begin{array}{llll}
1 & 0 & 0 & 0 \\
0 & 1 & 0 & 0 \\
0 & 0 & 1 & 0 \\
0 & 0 & 0 & 0
\end{array}\right)
$$

Since the differential operators in Eq. (3.11) do not depend on $\theta$, the solution must be an eigenfunction of the form $\hat{\mathbf{q}}_{0}=A(\theta) \boldsymbol{\Phi}(\theta, \eta)$. The unknown function $A(\theta)$ can be found from the Fredholm alternative theorem applied to (3.12) leading to the condition

$$
\int_{0}^{\infty} \boldsymbol{\Phi}^{+*} \mathcal{H}[A(\theta) \boldsymbol{\Phi}] \mathrm{d} \eta=0
$$

where $\boldsymbol{\Phi}^{+*}$ is the complex conjugate of the mode associated to the adjoint operator of $(3.13)$

$$
\mathcal{L}^{+}=\left[i \omega-i \alpha U-i \beta V-\frac{1}{\epsilon \operatorname{Re}_{s}}\left(\frac{\partial^{2}}{\partial \eta^{2}}-\alpha^{2}-\beta^{2}\right)\right] \mathbf{Q}-\left(\begin{array}{cccc}
0 & 0 & 0 & i \alpha \\
0 & 0 & 0 & i \beta \\
-\frac{\partial U}{\partial \eta} & -\frac{\partial V}{\partial \eta} & 0 & \frac{\partial}{\partial \eta} \\
i \alpha & i \beta & \frac{\partial}{\partial \eta} & 0
\end{array}\right) .
$$

Equation (3.16) can be written in the form

$$
\frac{\mathrm{d} A}{\mathrm{~d} \theta} \int_{0}^{\infty} \boldsymbol{\Phi}^{+*} \mathcal{H}_{1} \boldsymbol{\Phi} \mathrm{d} \eta+A \int_{0}^{\infty} \boldsymbol{\Phi}^{+*} \mathcal{H} \boldsymbol{\Phi} \mathrm{d} \eta=0,
$$

where the operator $\mathcal{H}_{1}$ is defined as

$$
\mathcal{H}_{1}=\left[U-\frac{2 i \alpha}{\epsilon \mathrm{Re}_{s}}\right] \mathbf{Q}+\left(\begin{array}{cccc}
0 & 0 & 0 & 1 \\
0 & 0 & 0 & 0 \\
0 & 0 & 0 & 0 \\
1 & 0 & 0 & 0
\end{array}\right)
$$

Equation (3.18) is a linear first-order homogeneous equation, so that the function $A(\theta)$ is obtained as

where

$$
A(\theta)=A\left(\theta_{0}\right) \exp \left[-\int_{\theta_{0}}^{\theta} \frac{N\left(\theta^{\prime}\right)}{M\left(\theta^{\prime}\right)} \mathrm{d} \theta^{\prime}\right]
$$

$$
M(\theta)=\int_{0}^{\infty} \boldsymbol{\Phi}^{+*} \mathcal{H}_{1} \boldsymbol{\Phi} \mathrm{d} \eta \quad, \quad N(\theta)=\int_{0}^{\infty} \boldsymbol{\Phi}^{+*} \mathcal{H} \boldsymbol{\Phi} \mathrm{d} \eta .
$$

\subsection{Numerical solution of the stability equations}

The stability equations are here solved in a three-step process. In the first step the surface of the sphere is subdivided into a number of stations in the azimuthal direction and the parallel eigenvalue problem (3.11) is solved at each of them: the equations are solved in primitive variables by using a Galerkin projection in terms of Chebyshev polynomials in the wall-normal direction. The use of the Chebyshev polynomials ensures a higher accuracy compared to standard finite-difference methods with a similar discretisation. An exponential map is adopted to map the Gauss-Lobatto grid points, used for the Chebyshev polynomials, into the physical space: 100 points are therefore distributed between the sphere surface $\eta=0$ and the top of the domain, $\eta_{\max }=20$. The stability equations are written and solved in primitive variables at all the collocation points but 
the ones at the boundaries $\left(\eta=0\right.$ and $\left.\eta=\eta_{\max }\right)$, where the boundary conditions $\hat{u}(0)=\hat{v}(0)=\hat{w}(0)=\hat{w}^{\prime}(0)=0$ and $\hat{u}\left(\eta_{\max }\right)=\hat{v}\left(\eta_{\max }\right)=\hat{w}\left(\eta_{\max }\right)=\hat{p}\left(\eta_{\max }\right)=0$ are enforced.

In the second step, the adjoint problem $\mathcal{L}^{+} \mathbf{q}_{0}^{+}=\mathbf{0}$ is solved at the same azimuthal stations (and the same boundary conditions). This allows the determination of the adjoint eigenfunctions to be used in the solvability condition (3.16). The third step consists of the calculation of the functions $A(\theta), M(\theta)$ and $N(\theta)$ by means of (3.20) and (3.21), where the operators $\mathcal{H}$ and $\mathcal{H}_{1}$ are given by (3.14) and (3.19), respectively. By means of the parallel eigenfunctions, $\boldsymbol{\Phi}_{0}$, determined at the first step and of the function $A$, the non-parallel eigenfunction is obtained through $\hat{\mathbf{q}}_{0}=A(\theta) \Phi(\theta, \eta)$.

\section{Results of the stability analysis}

Following Eq. (3.7), we interpret the perturbation system as having three control parameters $\operatorname{Re}=\operatorname{Re}_{s}^{1 / 2}$ (here preferred to facilitate the comparison with the literature on the rotating disk and sphere), $\bar{n}=n / \epsilon$ and $\omega$. Each parameter triple can be interrogated to determine its stability characteristics. We follow Garrett \& Peake (2002) by insisting that the disturbances rotate at some fixed multiple, $c$, of the surface speed of the sphere at all latitudes in this fixed frame of reference. That is, we equate the disturbance phase velocity $\omega / n$ to $c \sin \theta$. This then defines $\omega$ in terms of $\bar{n}$, leading to the new parameter triple $(\operatorname{Re}, \bar{n}, c)$. Recall that Re can be interpreted as the spin rate of the sphere while $\bar{n}$ is the azimuthal mode number of the disturbance under investigation (i.e. the number of vortices around the azimuth) and is necessarily an integer.

We proceed to consider the local stability properties of the flow incorporating nonparallel effects (i.e. without spatial homogenisation). The results of this analysis are associated with the appearance of spiral vortices within the boundary layer and can be related to the prior study of Garrett \& Peake (2002). We will discuss comparisons between our new results and those prior results as appropriate. However, when making such comparisons, it is important to note two significant distinctions: we now account for non-parallel effects, and our basic flow now allows for a better account of the boundarylayer eruption at the equator and also all 1/Re effects that were previously neglected. Unless otherwise stated, it should be assumed that all results are reported with both new aspects considered. Where necessary, we will occasionally report results from the non-parallel analysis performed on the previous steady flows (i.e. without the eruption and 1/Re effects), henceforth referred to as the Banks profiles (Banks 1965).

The growth rate of disturbances in the previous parallel-flow analysis (i.e. with spatial homogenisation) of Garrett \& Peake (2002) was given by $-\alpha_{i}$ and their strictly-local analysis required the stability to be determined separately at each latitude $\theta$. In contrast, here we will determine the stability properties over the entire flow field in each calculation. Furthermore, the allowance for non-parallel effects now means that the growth rate of disturbances is given by

$$
\sigma=-\alpha_{i}+\frac{\partial}{\partial \theta} \ln \sqrt{E}
$$

where $E$ is the disturbance kinetic energy given by

$$
E=\int_{0}^{\infty}\left(|\hat{u}|^{2}+|\hat{u}|^{2}+|\hat{u}|^{2}\right) \mathrm{d} \eta .
$$

As will be discussed in Section 5, the perturbation velocities account for $A(\theta)$, the correction for spatial inhomogeneity in $\theta$. The term $\partial \ln \sqrt{E} / \partial \theta$ is henceforth referred to as the non-parallel correction. In order to focus on the boundary layer, rather than the 


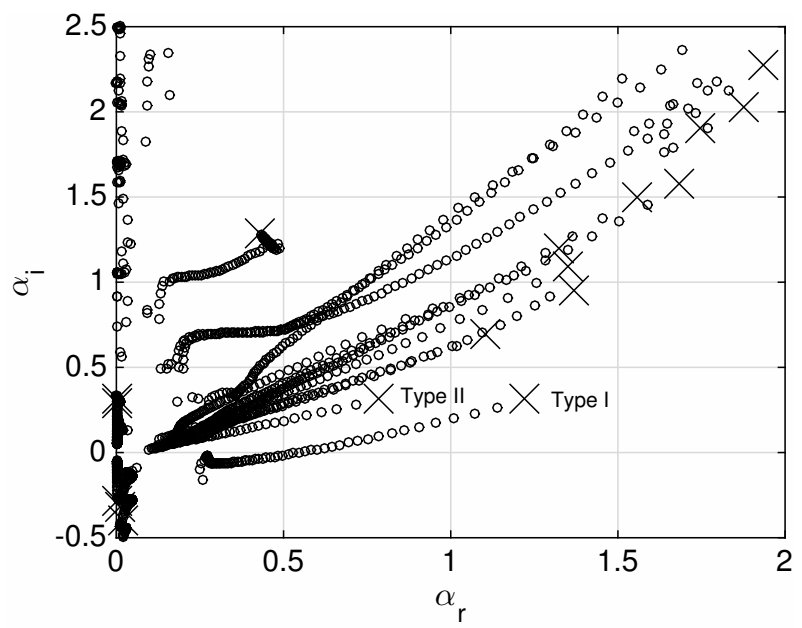

Figure 12: Eigenvalues' evolution at $(\operatorname{Re}, \bar{n}, c)=(600,30,1)$. Crosses indicate the eigenvalues at $\theta=1^{\circ}$ and the circles show their evolution as $\theta$ steps towards the equator in unit increments (right to left).

erupting jet at the equator, the stability results are only considered over the latitudinal domain between the pole and $\theta=80^{\circ}$.

The perturbation system derived in Section 3 is found to permit a number of distinct eigenmodes at each parameter triple $(R e, \bar{n}, c)$. This is illustrated in Figure 12 for the particular case with $(\operatorname{Re}, \bar{n}, c)=(600,30,1)$. Further investigation across the entire parameter space reveals that only a small number of these modes are physical (as evidenced by the form of the eigenfunctions, see Section 5) and only two can lead to instability. The appearance of two unstable modes is consistent with Garrett \& Peake (2002) on the rotating sphere and indeed with the broader literature on the rotating-disk system (Hall 1986; Malik 1986; Lingwood 1995). As will be discussed in the following sections, the mode denoted Type $I$ in the figure is found to be unstable over a much larger parameter space and is deemed equivalent to Garrett \& Peake's Type I mode. The Type I mode is known to arise from inviscid cross-flow effects and leads to the vortices observed in experiments. It is also related to the appearance of a local absolute instability. The Type II mode is known to arise from viscous effects and is found to be unstable within a relatively limited parameter space. The Type II mode is of theoretical rather than practical interest and we will focus on the Type I mode in what follows. The Type II mode is however considered separately in Sections 4.2 and 5.2 .

\subsection{The Type I mode}

\subsubsection{Stationary disturbances}

We begin with the study of Type I stationary disturbances, that is, disturbances that rotate with the local surface of the sphere, and fix $c=1$. This results in the parameter pair $(R e, \bar{n})$ giving the sphere spin rate and mode number of interest, respectively. Experiments (Sawatzki 1970; Kohama \& Kobayashi 1983) report the appearance of such stationary vortices on all but highly-polished surfaces and this is an appropriate assumption to base the majority of our analysis. Non-stationary modes (namely with $c \neq 1$ ) are considered in Section 4.1.2.

Figure 13 shows the parallel growth rate, $\sigma$, at $\operatorname{Re}=160$ and $\operatorname{Re}=700$ as a function of latitude with and without the non-parallel correction for various values of $\bar{n}$. The results 
for $\operatorname{Re}=160$ show a significant discrepancy arising from the parallel and non-parallel flow assumptions. This is in contrast to $\mathrm{Re}=700$ where the non-parallel correction is seen to be relatively small, particularly for larger $\bar{n}$. These observations are consistent with Garrett \& Peake's assertion that the non-parallel correction reduces with increasing Re.

In each figure the appearance of neutral growth, $\sigma=0$, is indicated with a circle. This can be interpreted as the latitudinal location of the onset of the Type I instability for the particular mode number, $\bar{n}$, at spin rate $\operatorname{Re}$. At $\operatorname{Re}=160$ we see that the $\bar{n}=9$ mode leads to a small pocket of instability at latitude $\theta=74^{\circ}$. The other mode numbers shown at this Reynolds number are seen to be stable and it is clear that $R e=160$ is close to the critical Reynolds number for onset of Type I instability over the sphere. In contrast, at $\mathrm{Re}=700$ all three mode numbers illustrated are unstable. In particular, the mode with $\bar{n}=20$ is unstable beyond $\theta=22^{\circ} ; \bar{n}=40$ beyond $\theta=30^{\circ}$; and $\bar{n}=60$ beyond $\theta=43^{\circ}$.

Such sampling of the stability of the flow can be performed at each parameter pair $(\mathrm{Re}, \bar{n})$ and this approach can be used to generate a single neutral curve for stationary Type I modes across the entire system. Figure 14 shows this neutral curve in the $\operatorname{Re}^{2}-\bar{n}$ planef. Here the squares indicate neutrally unstable parameter pairs and a cross indicates the most amplified mode at each Re. The resulting neutral curve is single-lobed and shows a critical Reynolds number of $\operatorname{Re}_{c, I}=159$, below which no Type I modes are predicted to be unstable. Note that $\operatorname{Re}_{c, I}$ is achieved for $\bar{n}=10$. Furthermore, the dotted line with circles shows experimental observations as reported by Kohama \& Kobayashi (1983) for a sphere with diameter $D=250 \mathrm{~mm}$. We see remarkable agreement between these experimental observations and the predicted maximum amplified mode at each Re.

Figure 15 presents the same unstable points given in Figure 14 but in terms of the $\operatorname{Re}^{2}-\theta$ plane. In this context $\theta$ is the location of the appearance of neutral stability, that is, locations analogous to those shown with circles in Figure 13. Four independent experimental data sets are available in this form, each from different sized spheres (Sawatzki 1970; Kohama \& Kobayashi 1983), and these are also shown in the figure. The experimental comparisons with the most amplified mode numbers are less remarkable than in Figure 14. However, we do note that, with the exception of Kohama \& Kobayashi's outlying results for the very small sphere (diameter $D=70 \mathrm{~mm}$ ), each experimental data set falls within our predicted region of instability.

\subsubsection{Travelling disturbances}

By varying the value of $c$ we are able to consider Type I disturbances that are moving relative to the sphere surface. The case that $c>1$ denotes disturbances travelling more quickly than the local surface speed, and $c<1$ denotes disturbances travelling more slowly. For example, $c=1.1$ and $c=0.9$ would correspond to disturbances travelling at $110 \%$ and $90 \%$ of the local surface speed, respectively. In practice, travelling vortices are difficult to observe in experiments unless great care is taken to avoid the appearance of any surface roughness (that would otherwise fix the vortex pattern relative to the rotating surface) and the boundary layer excitation results instead from free-stream turbulence. However, in theory, travelling modes can be even more dangerous than stationary modes (in the sense of lower critical Re) and are also known to play a role in local absolute instability (see, for example, the work of Lingwood 1995; Corke \& Knasiak 1998; Othman \& Corke 2006; Corke et al. 2007, on the related rotating-disk system).

$\dagger$ Note that neutral curves are presented in terms of the spin Reynolds number, $\operatorname{Re}_{s}=\operatorname{Re}^{2}$ which is typically used to report experimental data and its use here permits direct comparisons between our predictions and published experimental measurements. 


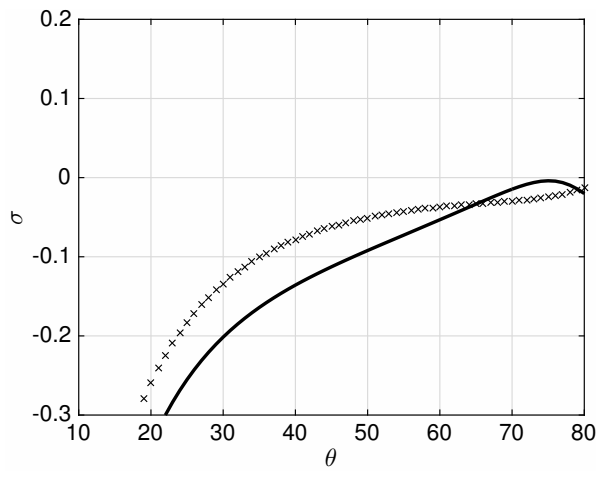

(a) $\operatorname{Re}=160, \bar{n}=7$.

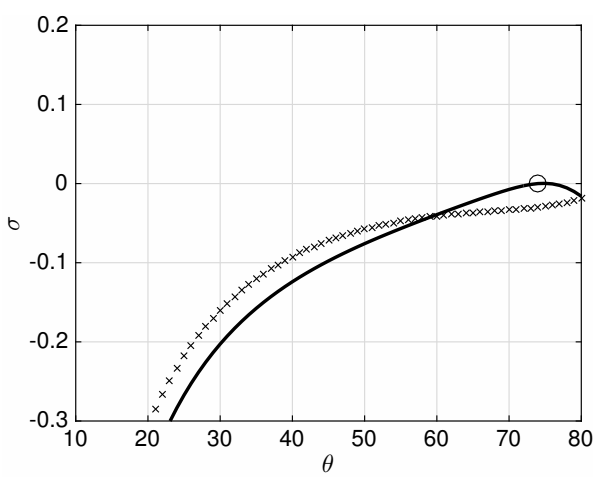

(c) $\operatorname{Re}=160, \bar{n}=9$.

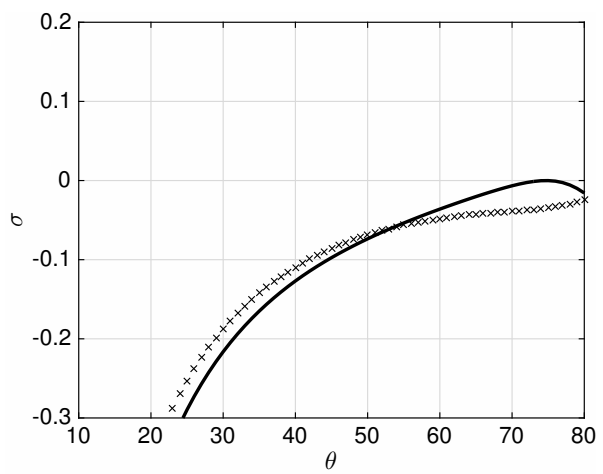

(e) $\operatorname{Re}=160, \bar{n}=11$.

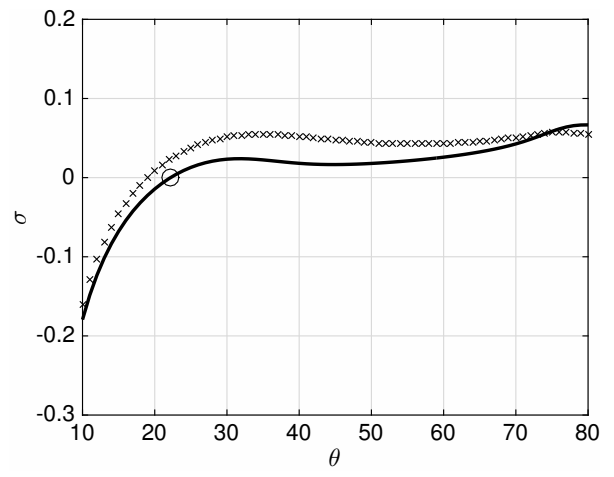

(b) $\operatorname{Re}=700, \bar{n}=20$.

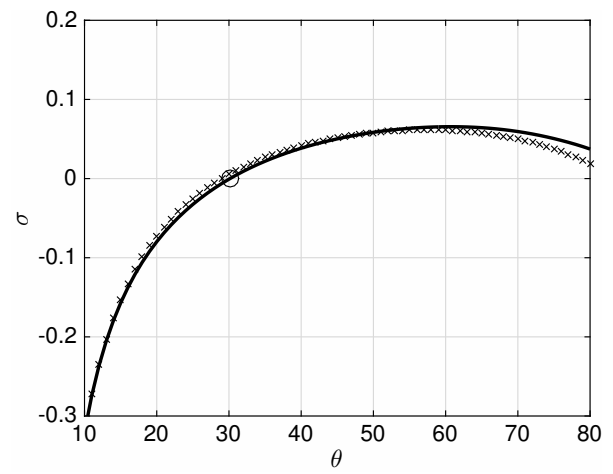

(d) $\operatorname{Re}=700, \bar{n}=40$.

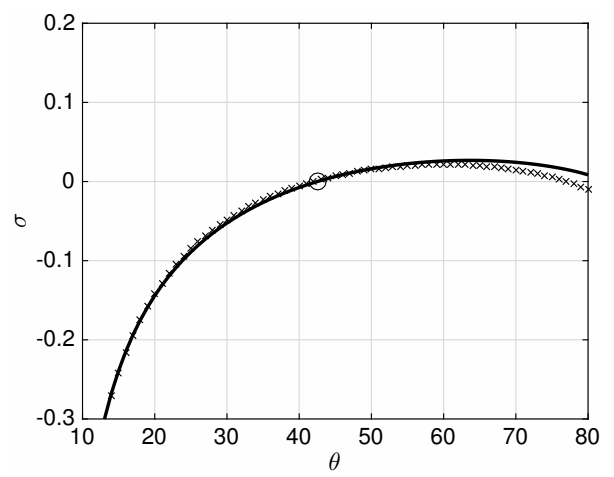

(f) $\operatorname{Re}=700, \bar{n}=60$.

Figure 13: Local growth rates at various $\operatorname{Re}$ and $\bar{n}$; non-parallel growth $(\sigma$, solid lines) and parallel growth $\left(-\alpha_{i}\right.$, crosses $)$. Circles indicate neutral $\sigma$.

The approach taken in Section 4.1.1 has been repeated for various $c$ and Table 1 gives the resulting mode number for the lowest critical Re in each case. We see that slowly travelling modes are predicted to be less dangerous (i.e. increased $\operatorname{Re}_{c}$ ) with reduced $c$. In contrast, quickly travelling modes are predicted to be more dangerous with increased $c$. Note however that the corresponding mode number reduces to small values that are unphysical in practice - certainly the experimental observations of Sawatzki (1970) and Kohama \& Kobayashi (1983) never report fewer than ten vortices. 


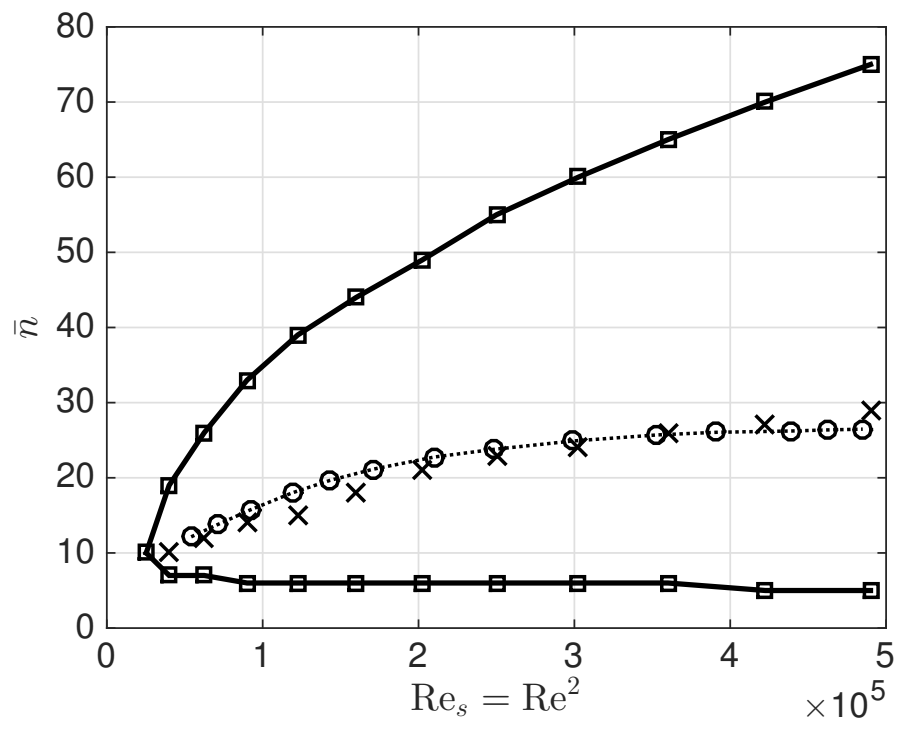

Figure 14: Comparison of the neutral curve for the stationary Type I mode (solid line with squares) in the $\operatorname{Re}_{s}-\bar{n}$ plane with the experimental data of Kohama \& Kobayashi (1983) for $D=250 \mathrm{~mm}$ (dotted line with circles). Crosses denote the mode number with the maximum amplification at each Re.

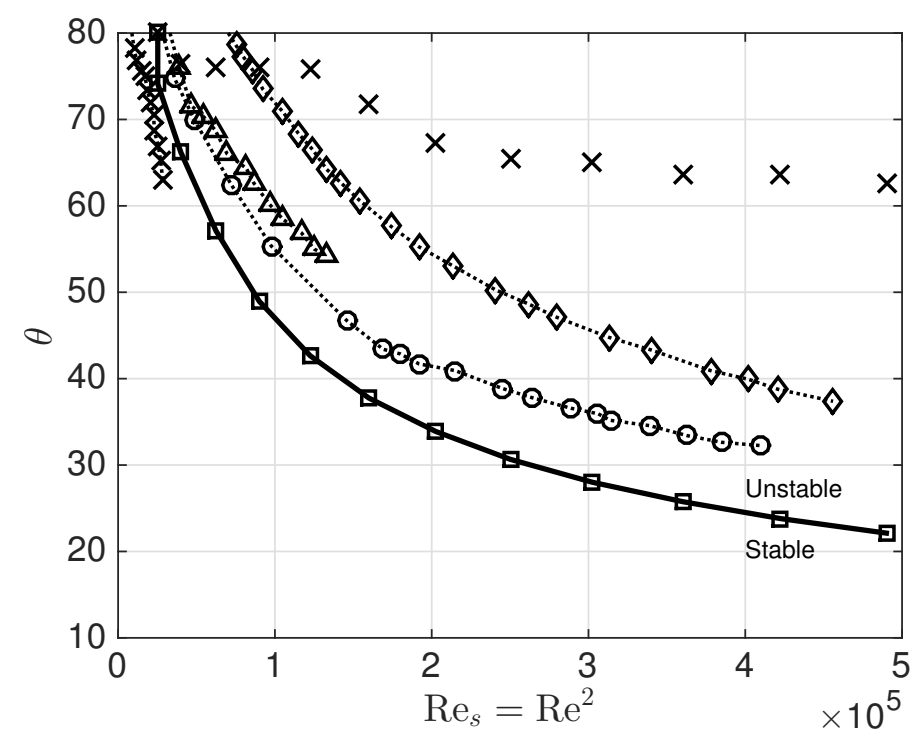

Figure 15: Comparisons of the neutral curve for the stationary Type I mode (solid line with squares) in the $\operatorname{Re}_{s}-\theta$ plane with experimental data of Kohama \& Kobayashi (1983) $D=70 \mathrm{~mm}$ (dotted line with crosses); $D=150 \mathrm{~mm}$ (dotted line with triangles) $D=$ $250 \mathrm{~mm}$ (dotted line with circles); and Sawatzki (1970), D = $240 \mathrm{~mm}$ (dotted line with diamonds). Isolated crosses denote the latitude at the onset of the most amplified mode at each Re. 


\begin{tabular}{c|ccccccccc}
$c$ & 1.5 & 1.4 & 1.3 & 1.2 & 1.1 & 1 & 0.9 & 0.8 & 0.7 \\
\hline$R e_{c}$ & 135 & 137 & 141 & 145 & 151 & 159 & 171 & 194 & 253 \\
$\bar{n}$ & 5 & 6 & 7 & 7 & 8 & 10 & 12 & 15 & 24
\end{tabular}

Table 1: The critical Re and associated mode number for the onset of travelling disturbances at various $c$.

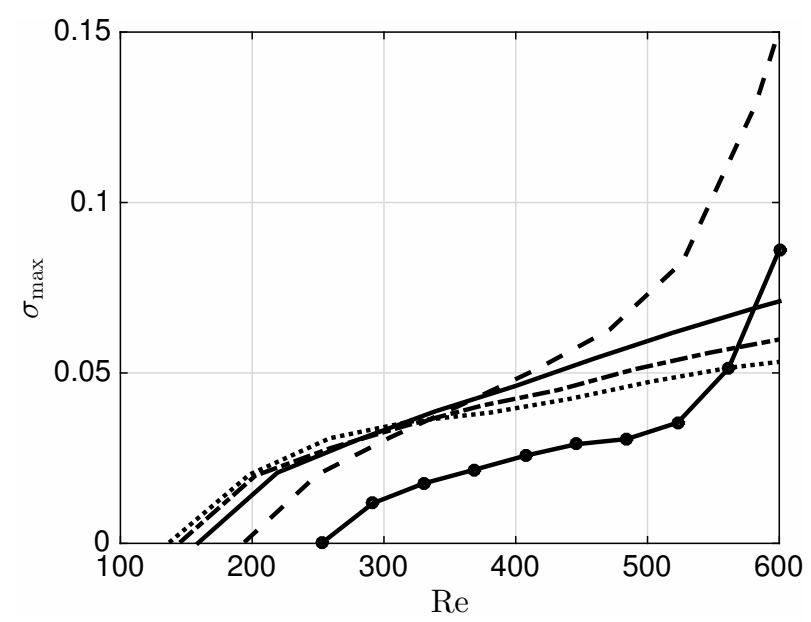

Figure 16: Evolution of $\sigma_{\max }$ for $c=0.8$ (dashed line), 1 (solid line), 1.2 (dash-dot line), 1.4 (dotted line) and 0.7 (line with circles).

Figure 16 shows the evolution of the maximum amplification rate, $\sigma_{\max }$, of each travelling Type I mode as Re is increased into the locally unstable regime. Amplification rates for very slowly travelling modes, that is with $c \leqslant 0.7$, are found to be significantly lower than the more rapidly travelling modes for moderate Re. The figure suggests that stationary and quickly travelling modes dominate for $\operatorname{Re}<400$. However, despite the mode with $c=0.8$ having a later onset, it is the most amplified by some margin for $\operatorname{Re}>400$. We note that these observations are consistent with Garrett (2010) who found that the Type I mode with $c=0.75$ has the greatest amplification rate under the parallel-flow assumption. There he associates this result with the single experimental observation by Kobayashi \& Arai (1990) of vortices travelling at $76 \%$ of the local surface speed of the sphere rotating in otherwise still fluid. Unfortunately no further information is given by Kobayashi \& Arai and further comparisons with our new theoretical prediction cannot be made.

\subsection{Stationary Type II modes and the full non-parallel neutral curve}

As illustrated in Figure 12, our analysis leads to the prediction of an additional instability mechanism that we have associated with the Type II mode identified by the parallel-flow study of Garrett \& Peake (2002). Although there is no experimental evidence for the Type II mode in the literature for the rotating sphere or related boundary-layer flows, predictions of its theoretical existence provide an additional means by which to compare across analytical studies. Figure 17 illustrates the non-parallel growth rates of this mode 


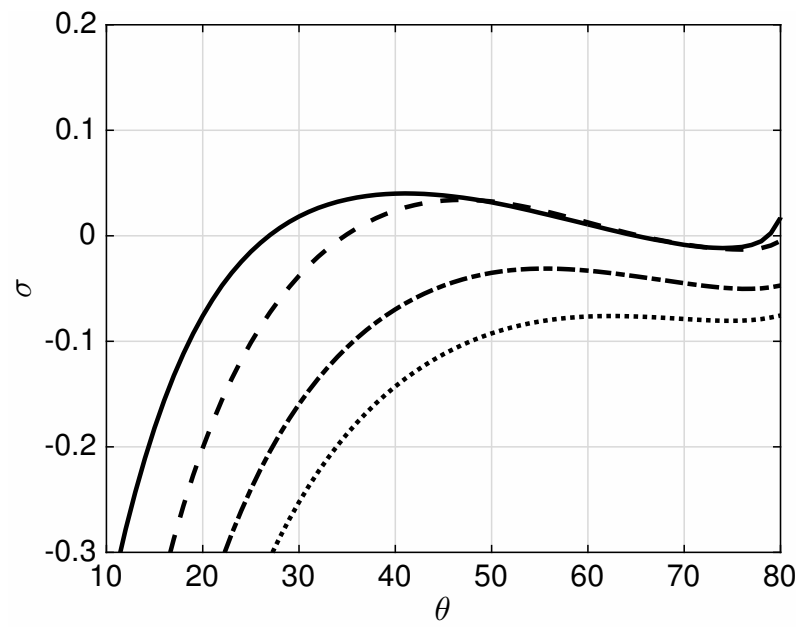

Figure 17: Growth rates $\sigma$ for stationary Type II modes at Re $=50$ for $\bar{n}=1$ (solid line), 2 (dashed line), 3 (dash-dot line) and 4 (dotted line).

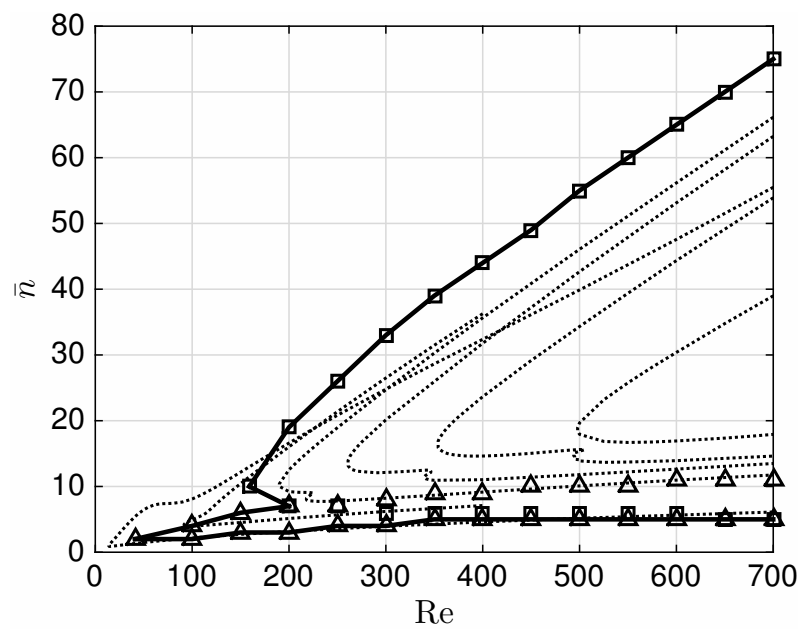

Figure 18: The full neutral curve for stationary $(c=1)$ Type I (squares) and Type II (triangles) modes. The dotted lines are the strictly local neutral curves due to Garrett $\&$ Peake (2002) at $\theta=30^{\circ}-80^{\circ}$ in ten degree increments (right to left).

at $\operatorname{Re}=50$ for increasing $\bar{n}$ and pockets of instability can be seen clearly for $\bar{n}=1$ and 2 .

Following the process discussed in Section 4.1.1, it is possible to examine the stability of the Type II mode at each parameter triple $(\operatorname{Re}, \bar{n}, c)$ and generate a neutral curve for this mode. This has been done for stationary disturbances $(c=1)$ and the full neutral curve arising from the Type I and Type II modes is shown in the Re- $\bar{n}$ plane in Figure 18. Note that the familiar two-lobed structure is recovered. That is, the Type II mode has led to an additional lobe in the neutral curve characterised by small $\bar{n}$ that is seen to be unstable at all Re above a critical value of $\operatorname{Re}_{c, I I}=41$. The critical Reynolds number for the onset of the Type II mode is therefore considerably lower than that for the Type I mode computed as $\operatorname{Re}_{c, I}=159$ in Section 4.1.1. 
The strictly local analysis of Garrett \& Peake (2002) generates a different neutral curve at each latitudinal position. Our single neutral curve for the entire flow field should therefore be compared to the envelope of all neutral curves generated by Garrett \& Peake and this comparison is shown in Figure 18. The comparison shows broad qualitative agreement, however there are some quantitative differences between the two studies. The most significant being around the predicted critical Reynolds numbers of each mode and along the upper branch of the Type I lobe. Garrett \& Peake report that their Type II mode dominates for latitudes above $\theta=66^{\circ}$ and so the comparison of the Type I unstable points is relevant for all but the two leftmost curves in Figure 18 (where $\theta=70^{\circ}$ and $80^{\circ}$ ). With this is mind we see good agreement between the critical Reynolds numbers for the onset of their Type I mode, elsewhere reported to be Re $=155$ (Garrett 2002), and our $\operatorname{Re}_{c, I}=159 \dagger$. In terms of the Type II mode, Garrett \& Peake report a critical Reynolds number of around $\operatorname{Re}=14$ for the neutral curve at $\theta=80^{\circ}$. This compares poorly with our critical value of $\operatorname{Re}_{c, I I}=41$. However, as discussed in Section 4.1, discrepancies at these very low Reynolds numbers are likely to be due to the parallel-flow assumption used by Garrett \& Peake which is now known to be significant at low Re, in addition to the fact that their base flow was different.

Although not shown here, the neutral curve resulting from our non-parallel analysis of the Banks flow profiles shows very good agreement with Garrett \& Peake along the upper neutral curve. We therefore attribute the difference along the upper branch to the allowance for the boundary-layer eruption and other $1 /$ Re effects in this new analysis. It is interesting to note that the close agreement shown between theory and experiment in Figure 14 is lost when using the Banks profiles; in particular, the number of vortices is then consistently under-predicted when compared to experimental data.

\section{Eigenfunctions}

We now turn to a discussion of the eigenfunctions of the system. In particular, we focus on the azimuthal perturbation velocities, i.e. $\hat{\phi}(\eta, \theta)=\hat{v}(\eta, \theta)$ in the notation of Eq. (3.7). Note that our choice of the azimuthal perturbation component is arbitrary and any perturbation component (velocity or pressure) could be used.

Within our formulation the parallel-flow eigenfunction, $\bar{v}(\eta, \theta)$, is computed together with its non-parallel correction, $A(\theta)$, and then combined to obtain the non-parallel eigenfunction at each parameter triple $(\operatorname{Re}, \bar{n}, c)$,

$$
\hat{v}(\eta, \theta)=\bar{v}(\eta, \theta) A(\theta) .
$$

Consistent with Cooper et al. (2015) and others in the literature we report the absolute value of the complex eigenfunctions in what follows.

As previously discussed, many of the additional modes identified in Figure 12 can be dismissed as numerical artefacts and these are identified by the unphysical shape of their parallel eigenfunction, $\bar{v}(\eta, \theta)$, sampled at some $\theta$. Example parallel Type I and Type II eigenmodes are illustrated in Figure 19.

\subsection{Spatial evolution of the Type I modes}

The behaviour of the Type I eigenfunction is found to be consistent across the entire parameter space and, by way of illustration, we present only the parameter triple $(\operatorname{Re}, \bar{n}, c)=(600,30,1)$. This particular parameter set is known to be unstable via the

$\dagger$ Note that Figure 18 does not show Garrett \& Peake's parallel-flow results at $\theta=66^{\circ}$, only at $\theta=60^{\circ}$ and $70^{\circ}$. 

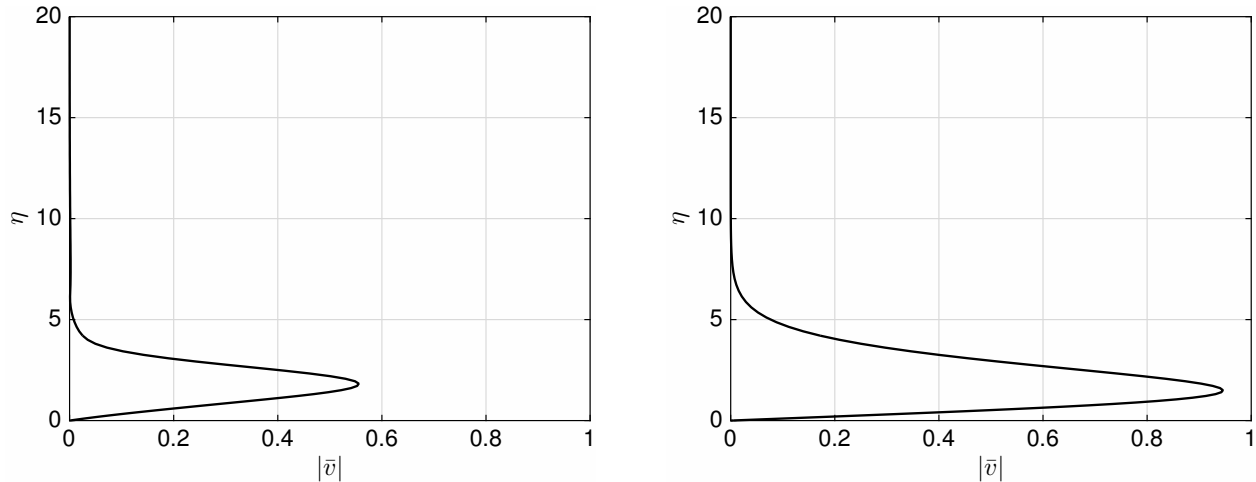

Figure 19: Physical eigenfunctions at $\theta=50^{\circ}$. (a) Type $\mathrm{I}|\bar{v}(\eta, 50)|$ for $(\operatorname{Re}, \bar{n}, c)=$ $(600,30,1)$, (b) Type II $|\bar{v}(\eta, 50)|$ for $(\operatorname{Re}, \bar{n}, c)=(100,4,1)$. (Each is normalised by $\max |\bar{v}(\theta, \eta)|$ at the particular $(\operatorname{Re}, \bar{n}, c)$.)
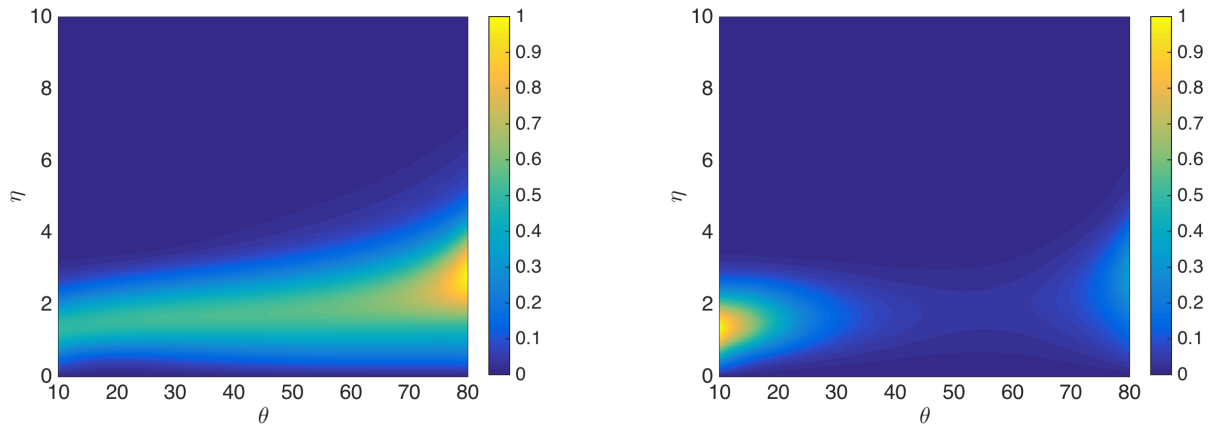

Figure 20: The Type I azimuthal perturbation velocity field for $(\operatorname{Re}, \bar{n}, c)=(600,30,1)$. (a) Parallel eigenfunction, $|\bar{v}(\theta, \eta)|$, (b) Non-parallel eigenfunction, $|\hat{v}(\theta, \eta)|$. (Each figure is normalised independently by its maximum value.)

Type I mode, as can be confirmed from Figure 18. The parallel eigenfunction, $\bar{v}(\theta, \eta)$, is shown in Figure 20(a) and that allowing for the non-parallel correction, $\hat{v}(\theta, \eta)$, is shown in Figure 20(b). The parallel eigenfunction shows that the Type I mode exists at all latitudes over the sphere and tends to grow in its normal extent and magnitude as the disturbance moves from the pole towards the equator. The non-parallel correction is seen to be significant and suggests that an unstable Type I mode would grow rapidly from even small latitudes.

\subsection{Spatial evolution of the Type II modes}

The parallel and non-parallel Type II eigenfunctions are illustrated from Figure 21 for $(\operatorname{Re}, \bar{c})=(100,4,1)$. This particular mode is known to be unstable, as can be confirmed in Figure 18. Consistent with the parallel Type I mode in Figure 20(a), the parallel Type II mode is seen to exist at all latitudes, however its normal extent and magnitude are reasonably constant over $\theta$. Furthermore, in direct contrast to the Type I mode, the non-parallel correction is seen to focus the importance of the Type II mode to higher latitudes. The contrasting behaviour of the non-parallel Type I and Type II modes is consistent with the conclusion of Garrett \& Peake that an unstable Type II mode is 

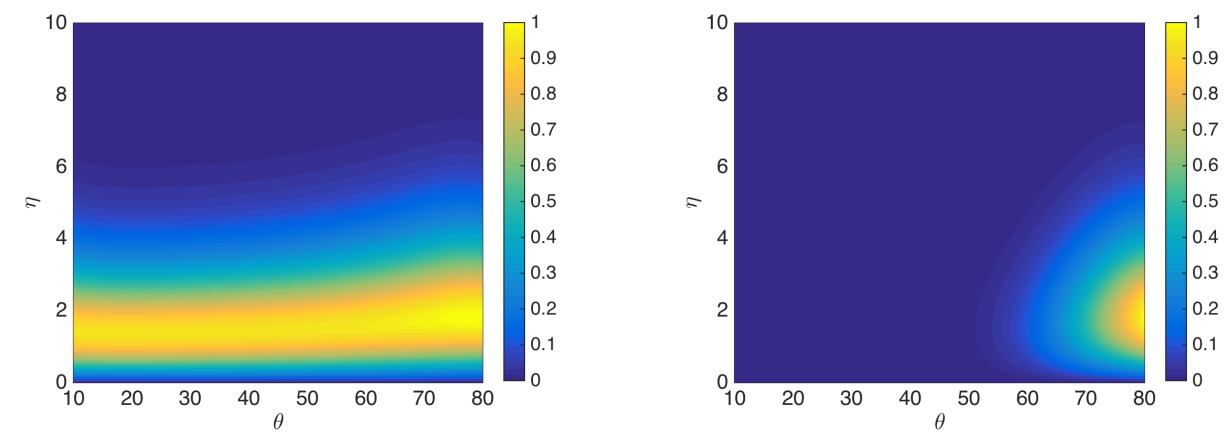

Figure 21: The Type II azimuthal perturbation velocity field for $(\operatorname{Re}, \bar{n}, c)=(100,4,1)$. (a) Parallel eigenfunction, $|\bar{v}(\theta, \eta)|$, (b) Non-parallel eigenfunction, $|\hat{v}(\theta, \eta)|$. (Each figure is normalised independently by its maximum value.)

potentially important only at higher latitudes, by which point unstable Type I modes would dominate the flow.

Note that each subfigure in Figures 20 and 21 is normalised by its own maximum value and quantitative comparisons should not be made between any plots.

\section{Conclusion}

We have considered the boundary-layer flow over the rotating sphere, both in terms of the computation of the steady flow and also its linear stability properties. The steadyflow solution has been improved significantly from the original formulation proposed by Howarth (1951) and solved by Banks (1965). It now better accounts for the boundarylayer eruption at the equator (Calabretto et al. 2015) and also the inclusion of a number of viscous terms that were previously neglected in the Howarth and Banks solutions.

The linear stability of the flow was considered as a function of spin rate (Reynolds number) using a weakly non-parallel analysis, that is, without imposing a spatial homogenisation of the boundary layer. As expected from prior parallel-flow studies (i.e. with spatial homogenisation imposed), two instability modes were found to dictate the local properties of the flow. These are denoted as the Type I and Type II modes. A single neutral curve was computed for the entire flow field and each mode was evident as a distinct lobe. The Type I mode is dominant and is associated with the experimentally observed spiral vortices in the boundary layer (Sawatzki 1970; Kohama \& Kobayashi 1983; Kobayashi \& Arai 1990). Stationary Type I and Type II modes were found to have critical Reynolds numbers of $\operatorname{Re}_{c, I}=159$ and $\operatorname{Re}_{c, I I}=41$, respectively. Remarkable agreement was found between the most amplified stationary Type I mode number and the experimentally observed number of vortices (Kohama \& Kobayashi 1983) as a function of spin rate. Furthermore, experimental observations of the latitudinal location of the onset of spiral vortices fell within our predicted neutral curve. Travelling Type I modes were also considered but are expected to correspond to the appearance of spiral vortices on only highly-polished surfaces, where the boundary layer excitation results from free-stream turbulence rather than surface roughness. In this case, Type I modes travelling with speeds of $70-80 \%$ of the local surface speed of the sphere were found to be the most amplified for sufficiently high spin rates. This is consistent with the experimental observation by Kobayashi \& Arai (1990) of "slow" vortices travelling at $76 \%$ of the local sphere surface speed. 
Parallel and non-parallel eigenmodes of both mode types have been computed for the first time. The spatial evolution of these suggests that, while the Type I mode is important at all latitudes, the Type II mode is potentially important at only larger latitudes, at which point unstable Type I modes would already dominate the flow. This is a potential explanation for why Type II modes have never been observed in experiments.

Our study extends those originally presented by Garrett \& Peake (2002) and Garrett (2010), performed using the parallel-flow approximation and Banks steady flow solutions. The non-parallel correction was found to be significant, particularly for low Reynolds numbers and small mode numbers.

SJG is supported by a Senior Research Fellowship of the Royal Academy of Engineering, funded by the Leverhulme Trust. The hospitality of Dr. Sharon Stephen and the wider Department of Mathematics, University of Sydney is acknowledged where SJG held a Visiting Professorship in 2016 and much of this paper was drafted. The continued hospitality of the Linné FLOW Centre, KTH is also acknowledged. Jacopo Canton and Dr. Philipp Schlatter are acknowledged for the fruitful discussions and for some simulations that supported the present mean-flow analysis.

\section{Appendix A. Double-deck description of the impinging region}

This section replicates the main steps of the derivation proposed by Smith \& Duck (1977) and extends it with the inclusion of the tangential velocity field. The derivation assumes a two-layer structure upstream of the separation point. In the upper layer the coordinates are scaled as

$$
\beta^{\prime}=\frac{\theta-\pi / 2}{\epsilon^{6 / 7}} \quad, \quad \eta=\frac{r-1}{\epsilon} .
$$

The velocity components are then linearised as

$$
\begin{aligned}
U=U_{0}\left(\frac{\pi}{2}, \eta\right)+\epsilon^{2 / 7} u_{1}, \quad V & =V_{0}\left(\frac{\pi}{2}, \eta\right)+\epsilon^{2 / 7} v_{1}, \\
W & =\epsilon^{3 / 7} w_{1}, \quad p=\epsilon^{4 / 7} p_{1},
\end{aligned}
$$

leading to the equations

$$
\begin{gathered}
\frac{\partial u_{1}}{\partial \beta^{\prime}}+\frac{\partial w_{1}}{\partial \eta}=0, \\
U_{0} \frac{\partial u_{1}}{\partial \beta^{\prime}}+w_{1} \frac{\partial U_{0}}{\partial \eta}=0, \\
U_{0} \frac{\partial v_{1}}{\partial \beta^{\prime}}+w_{1} \frac{\partial V_{0}}{\partial \eta}=0, \\
U_{0} \frac{\partial w_{1}}{\partial \beta^{\prime}}=-\frac{\partial p}{\partial \eta},
\end{gathered}
$$

with solution

$$
\begin{gathered}
u_{1}=A\left(\beta^{\prime}\right) \frac{\partial U_{0}}{\partial \eta} \quad, \quad v_{1}=A\left(\beta^{\prime}\right) \frac{\partial V_{0}}{\partial \eta} \quad, \quad w_{1}=-A^{\prime}\left(\beta^{\prime}\right) U_{0}\left(\frac{\pi}{2}, \eta\right), \\
p_{1}=P_{w}\left(\beta^{\prime}\right)+A^{\prime \prime}\left(\beta^{\prime}\right) \int_{0}^{\eta} U_{0}^{2}\left(\frac{\pi}{2}, \chi\right) \mathrm{d} \chi,
\end{gathered}
$$

for a certain unknown function $A\left(\beta^{\prime}\right)$. $A^{\prime}$ and $A^{\prime \prime}$ indicate the first and second derivative 


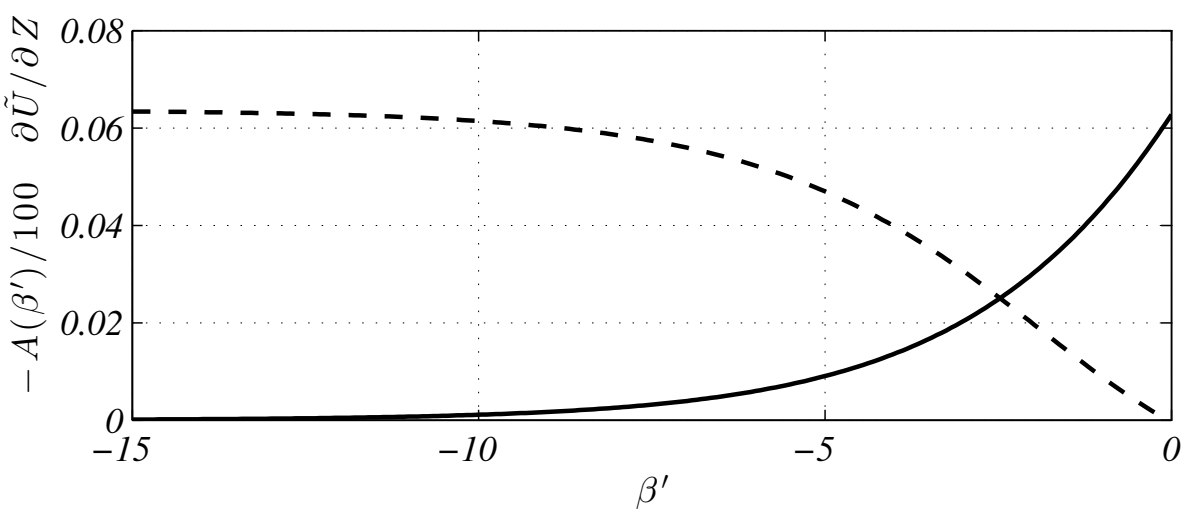

Figure 22: (Solid line) $-A / 100$, (dashed line) $\partial \tilde{U} / \partial Z$ upstream of the separation point.

of the $A$ function. As a boundary condition, $p_{1} \rightarrow 0$ as $\eta \rightarrow \infty$ leading to

$$
P_{w}\left(\beta^{\prime}\right)=-\frac{1}{\gamma} A^{\prime \prime}\left(\beta^{\prime}\right) \longrightarrow-\frac{\mathrm{d} P_{w}}{\mathrm{~d} \beta^{\prime}}=\frac{1}{\gamma} A^{\prime \prime \prime}\left(\beta^{\prime}\right),
$$

where $1 / \gamma=\int_{0}^{\infty} U_{0}^{2}(\pi / 2, \chi) \mathrm{d} \chi=0.0583$.

Since $u_{1} \rightarrow \kappa A$ as $\eta \rightarrow 0$ (with $\kappa$ indicating the first derivative of the $U_{0}$ profile at $\eta=0)$, a lower deck is needed. This is defined by the stretched variables $Z=\epsilon^{-9 / 7}(r-$ 1) $=\epsilon^{-2 / 7} \eta$, through the asymptotic expressions

$$
U=\epsilon^{2 / 7} \tilde{U} \quad, \quad V=1+\epsilon^{2 / 7} \tilde{V} \quad, \quad W=\epsilon^{5 / 7} \tilde{W},
$$

and by the equations

$$
\begin{gathered}
\frac{\partial \tilde{U}}{\partial \beta^{\prime}}+\frac{\partial \tilde{W}}{\partial Z}=0 \\
\tilde{U} \frac{\partial \tilde{U}}{\partial \beta^{\prime}}+\tilde{W} \frac{\partial \tilde{U}}{\partial \eta}=-\frac{\mathrm{d} P_{w}}{\mathrm{~d} \beta^{\prime}}+\frac{\partial^{2} \tilde{U}}{\partial Z^{2}}=\frac{1}{\gamma} A^{\prime \prime \prime}\left(\beta^{\prime}\right)+\frac{\partial^{2} \tilde{U}}{\partial Z^{2}}, \\
\tilde{U} \frac{\partial \tilde{V}}{\partial \beta^{\prime}}+\tilde{W} \frac{\partial \tilde{V}}{\partial \eta}=\frac{\partial^{2} \tilde{V}}{\partial Z^{2}},
\end{gathered}
$$

subjected to the boundary conditions

$$
\begin{gathered}
\tilde{U}=\tilde{V}=\tilde{W}=0 \quad \text { for } \quad Z=0, \\
\tilde{U} \rightarrow \kappa(Z+A) \quad, \quad \tilde{V} \rightarrow \xi(Z+A) \quad \text { as } \quad Z \rightarrow \infty,
\end{gathered}
$$

where $\xi$ indicates the first derivative of $V_{0}(\pi / 2, \eta)$ at $\eta=0$.

The function $A\left(\beta^{\prime}\right)$ is not determined from an explicit boundary condition to be fulfilled but it is here found as an eigensolution of the numerical problem (A 11-A 12), as discussed by Smith \& Duck (1977). Its initial assumed value determines the position of the separation point (here set at $\beta^{\prime}=0$ ). Figure 22 shows its distribution together with the shear stress (the same figure is reported by Smith \& Duck 1977), indicating that the effect of the separation is felt up to $\beta^{\prime} \approx-10$.

\section{REFERENCES}

Appelquist, E., Schlatter, P., Alfredsson, P. H. \& Lingwood, R. J. 2015 a Global linear 
instability of the rotating-disk flow investigated through simulations. J. Fluid Mech. 765, 612-631.

Appelquist, E, Schlatter, P, Alfredsson, P H \& Lingwood, R J $2015 b$ Investigation of the Global Instability of the Rotating-disk Boundary Layer. Procedia IUTAM 14, 321-328.

BAnks, W. H. H. 1965 The boundary layer on a rotating sphere. Quart. J. Mech. and Applied Math. 18, 443-454.

BANKs, W. H. H. \& ZATURSKA, M. B. 1979 The collision of unsteady laminar boundary layers. J. Eng. Math. 13, 193-212.

BARrow, A. \& Garrett, S. J. 2013 Convective and absolute instabilities in the boundary layer over rotating spheres with surface mass flux and incident axial flow. Eur. J. Mech. B/Fluids 38, 93-100.

Barrow, A., Garrett, S. J. \& Peake, N. 2014 Global linear stability of the boundary-layer flow over a rotating sphere. Eur. J. Mech. B/Fluids 49, 301-307.

Bowden, F. P. \& Lord, R. G. 1963 The aerodynamic resistance to a sphere rotating at high speed. Proc. R. Soc. Lond. A 271.

Calabretto, S. A. W., Levy, B., Denier, J. P. \& Mattner, T. W. 2015 The unsteady flow due to an impulsively rotated sphere. Proceedings of the Royal Society A: Mathematical, Physical and Engineering Science 471 (2181), 20150299-19.

Cooper, A. J., Harris, J. H., Garrett, S. J., Özkan, M. \& Thomas, P. J. 2015 The effect of anisotropic and isotropic roughness on the convective stability of the rotating disk boundary layer. Phys. Fluids 27 (1), 014107-17.

Corke, T.C., Matlis, E. H. \& Othman, H. 2007 Transition to turbulence in rotating-disk boundary layers-convective and absolute instabilities. J. Eng. Math. 57, 253.

Corke, T. C. \& KNASIAK, K. F. 1998 Stationary travelling crossflow mode interactions on a rotating disk. J. Fluid Mech. 355, 285.

Davies, C. \& CARPenter, P. W. 2003 Global behaviour corresponding to the absolute instability of the rotating-disc boundary layer. J. Fluid Mech. 486, 287-329.

Dennis, S. C. R. \& Duck, P. W. 1988 Unsteady flow due to an impulsively started rotating sphere. Computers \& Fluids 16, 291-310.

Dennis, S. C. R., Singh, S. N. \& Ingham, D. B. 1980 The steady flow due to a rotating sphere at low and moderate reynolds numbers. J. Fluid Mech. 101, 257-279.

Garrett, S. J. \& Peake, N 2004 The stability of the boundary layer on a sphere rotating in a uniform axial flow. Eur. J. Mech. B/Fluids 23 (2), 241-253.

Garrett, S. J. 2002 The Stability and Transition of the Boundary Layer on Rotating Bodies. PhD thesis, University of Cambridge.

Garrett, S. J. 2010 Vortex-speed selection within the boundary-layer flow over a rotating sphere placed in an enforced axial flow. Eur. J. Mech. B/Fluids 29, 84-92.

Garrett, S. J. \& Peake, N. 2002 The stability and transition of the boundary layer on a rotating sphere. J. Fluid Mech. 456, 199-218.

Gregory, N., Stuart, J. T. \& Walker, W. S. 1955 On the stability of three dimensional boundary layers with application to the flow due to a rotating disk. Phil. Trans. R. Soc. Lond. 248, 155-199.

HALL, P. 1986 An asymptotic investigation of the stationary modes of instability of the boundary layer on a rotating disc. Proc. R. Soc. Lond. A 406, 93-106.

Hollerbach, R., Wiener, R. J., Sullivan, I. S., Donnelly, R. J. \& C. F., Barenghi 2002 The flow around a torsionally oscillating sphere. Phys. Fluids 14, 4192.

Howarth, L. 1951 Note on the boundary layer on a rotating sphere. Phil. Mag. Ser. 42, 13081315.

Imayama, S., Alfredsson, P. H. \& Lingwood, R. J. 2012 A new way to describe the transition characteristics of a rotating-disk boundary-layer flow. Phys. Fluids 24 (3), 031701.

Imayama, S., Alfredsson, P. H. \& Lingwood, R. J. 2013 An experimental study of edge effects on rotating-disk transition. J. Fluid Mech. 716, 638-657.

Imayama, S., Alfredsson, P. H. \& Lingwood, R. J. 2014 On the laminar-turbulent transition of the rotating-disk flow: the role of absolute instability. J. Fluid Mech. 745, 132-163.

Kobayashi, R. \& ARAI, T. 1990 Spiral vortex behaviour in transition region and separation of three-dimensional boundary layers on spheres rotating in axial flow. In Laminar Turbulent Transition, IUTAM Symposium Toulouse (ed. D. Arnal \& R. Michel), pp. 551-557. 
Конама, O. \& Kobayashi, R. 1983 Boundary-layer transition and the behaviour of spiral vortices on rotating spheres. J. Fluid Mech. 137, 153-164.

Kundu, P. K., Cohen, I. M. \& Dowling, D. R. 2016 Fluid mechanics. Sixth edition: Elsevier.

Lingwood, R. J. 1995 Absolute instability of the boundary layer on a rotating disk. J. Fluid Mech. 299, 17-33.

MALIK, M. R. 1986 The neutral curve for stationary disturbances in rotating-disk flow. J. Fluid Mech. 164, 275-287.

Monkewitz, P. A., Huerre, P. \& Chomaz, J.-M. 1993 Global linear stability analysis of weakly non-parallel shear flows. J. Fluid Mech. 251, 1-20.

Othman, H. \& Corke, T. C. 2006 Experimental investigation of absolute instability of a rotating-disk boundary layer. J. Fluid Mech. 565, 63.

Phares, D. J., Smedley, G. T. \& Flagan, R. C. 2000 The inviscid impingement of a jet with arbitrary velocity profile. Phys. Fluids 12, 2046-2055.

PIER, B. 2003 Finite-amplitude crossflow vortices, secondary instability and transition in the rotating-disk boundary layer. J. Fluid Mech. 487, 315-343.

Riley, N. 1962 Radial jets with swirl. part i. incompressible flow. Quart. J. Mech. and Applied Math. XV, 435-458.

Rubel, A. 1983 Inviscid axisymmetric jet impingement with recirculating stagnation regions. AIAA J. 21, 351-357.

SAwatzKi, O. 1970 Flow field around a rotating sphere. Acta Mechanica 9 (3), 159-214.

Schwarz, W. H. 1963 The radial free jet. Chem. Eng. Sci. 18, 779-786.

Siddiqui, M. E., Mukund, V., Scott, J. \& Pier, B. 2013 Experimental characterization of transition region in rotating-disk boundary layer. Phys. Fluids 25 (3), 034102.

Simpson, C. J. \& Stewartson, K. 1982 A note on a boundary-layer collision on a rotating sphere. J. of Applied Mathematics and Physics (ZAMP) 33, 370-378.

Smith, F. T. \& Duck, P. W. 1977 Separation of jets or thermal boundary layers from a wall. Q. J. Mechanics Appl. Math. 30, 143-156.

Squire, H. B. 1955 Radial jets. In Fifty Years of Boundary Layer Research (ed. H. Görtler \& W. Tollmien). Vieweg.

Stewartson, K. 1958 On rotating laminar boundary layers. In Grenzschichtforschung / Boundary Layer Research (ed. H. Görtler). Springer-Verlag OHG.

VAn Dommelen, L. L. 1990 On the lagrangian description of unsteady boundary-layer separation. part 2. the spinning sphere. J. Fluid Mech. 210, 627-645. 